\title{
Low-Overhead Low-Complexity Carrier Phase Recovery Technique for Coherent Multi-Band OFDM-Based Superchannel Systems Enabled by Optical Frequency Combs
}

\author{
Faith K. Deynu 1,2 (D), Evans W. Akpari² (D) Constant Akama² (1) \\ ${ }^{1}$ University of Electronic Science \& Technology of China, School of Communication and Information \\ Engineering, Key Laboratory of Optical Fiber Sensing and Communications (Education Ministry of China), No. \\ 2006-Xiyuan Avenue-West Hi-Tech Zone, Chengdu, China, 611731.faithdeynu@yahoo.com, \\ ${ }^{2}$ Ho Technical University (HTU), Department of Electrical/Electronic Engineering (EEE), P.O. Box HP-217, \\ Ho, Ghana.akparievanswilson85@gmail.com,constantakama@gmail.com
}

\begin{abstract}
In this paper, we have designed a low-overhead lowcomplexity carrier phase tracking scheme for OFDM-based superchannel transmission system enabled by optical frequency combs. In this scheme, taking advantage of the broadband phase coherence provided by optical frequency combs among the OFDMbands, the carrier phase retrieved from pilot-subcarriers of the OFDM-band on the central wavelength channel is reused for the OFDM-bands on the other wavelength channels. In this case, the overall pilot-subcarrier overhead and DSP complexity is significantly reduced since the pilot-subcarriers occupy a small fraction of the overall OFDM bandwidth. The feasibility of this joint-carrier phase tracking scheme has been verified successfully via comprehensive simulation, where results show that the BER threshold for soft-decision FEC could be achieved for $50 \mathrm{GHz}-$ spaced 5-band 4-QAM, 8-QAM, 16-QAM and 32-QAM OFDMbased superchannel signals with zero guard-band and both laser and nonlinear phase noise effects after $7000 \mathrm{~km}, 4000 \mathrm{~km}, 3000 \mathrm{~km}$ and $2000 \mathrm{~km}$ SSMF transmission respectively. The simulation results show that there exist chromatic dispersion-induced differential phase offset among the OFDM-bands whose impact on joint-carrier phase tracking depends on the modulation format, channel count and fiber length. Finally, we demonstrate experimentally the feasibility performance of the designed masterslave carrier phase tracking technique for comb-based OFDMbased superchannels.
\end{abstract}

Index Terms - coherent detection optical fiber systems, comb-based multiband OFDM superchannel, master-slave joint-carrier phase tracking technique, laser and nonlinear phase noise effects.

\section{INTRODUCTION}

Historically, optical fiber transmission systems operated at the very limits of electronic technology with data rates far in excess of that which could be generated or sampled digitally [1]. In order to circumvent the limited speed of analogue and digital converters, superchannels via optical parallelism has become the enabling technology to improve the spectral efficiency (SE) and scale up the capacity of today's fiber transport networks in a cost-effective manner [2]-[4]. The term superchannel refers to 
a set of channels that are co-generated, co-transmitted and co-detected as a single entity [2]-[4]. In this context, ultra-high-speed superchannels may be basically obtained via digital subcarrier multiplexing (DSCM) [5], space division multiplexing (SDM, i.e., spatial superchannel) [4], [6], wavelength division multiplexing (WDM, i.e., spectral superchannel) [2] or a combination of these techniques (i.e., hybrid superchannel) [3]. In particular, coherent spectrally efficient superchannel can be created through wavelength multiplexing of either spectrally Nyquist-shaped single-carrier modulated signals [7]-[14] or orthogonal frequency division multiplexing (OFDM)-modulated signals [15]-[19]. However, carrier phase noise is a major problem in coherent optical transmission systems [20]. Particularly, due to its relatively longer symbol duration, coherent optical OFDM (CO-OFDM) systems are more susceptible to effects of the carrier phase noise, compared to conventional single carrier systems [21], [22]. This is because the phase noise in CO-OFDM systems manifests in two ways - a deterministic common phase error (CPE) that uniformly rotates the phase of all subcarriers, and intercarrier interference (ICI) caused by loss of orthogonality between the subcarriers [23], [24].

For this reason, pilot-aided carrier phase recovery (PA-CPR) algorithm is commonly used in COOFDM systems [25]. This carrier phase estimation technique takes advantage of the readily available multiple parallel subcarriers in OFDM systems to allocate few subcarriers with pilot-symbols at designated positions across the OFDM spectrum for the PA-CPR. Since it does not suffer phase ambiguities or cycle-slips, the pilot-subcarrier-aided phase estimation technique is very simple and robust against the phase noise. But PA-CPR reduces the net data-rate and SE of the system as a result of the pilot-subcarrier overhead. Therefore, to reduce the overhead, a family of blind phase noise estimation methods have been introduced for CO-OFDM systems to compensate either the CPE only [26]-[31] or both CPE and ICI [32]-[36]. Others also performed the phase estimation in two stages in order to avoid or reduce the pilot overhead in CO-OFDM systems [37]-[39]. Compared with the PACPR technique, these blind CPR methods will suffer from high computational complexity (CC) when applied to demodulate various OFDM bands within a WDM-based superchannel, where the CC may be scaled up by a factor $K$ ( $K$ is the number of bands within the entire superchannel spectrum). For convenience here, we refer to the WDM channels after OFDM modulation as bands.

Meanwhile, WDM-based superchannels can be generated by using discrete [7], [8], [15], [16] or phase-locked (i.e., optical frequency comb) laser sources [9]-[19]. For OFDM-based superchannel systems with phase-locked carriers, the subcarriers of OFDM across the different WDM channels are orthogonal to each other regardless of the M-QAM format used for the individual comb lines because the subcarriers are phase-locked to one laser [17]-[19]. The orthogonality among the OFDM subcarriers guarantee a zero linear inter-channel cross-talk (interference) penalty between adjacent sub-bands, which removes the need for frequency guard-bands that are necessary between the subchannels of comb-based Nyquist-WDM superchannel transmission systems [9]-[14]. In this case, each optical carrier can be seen as conveying a portion of the transmitted signal with strong overlapping between them, so that each OFDM-band can be perceived to be an orthogonal extension 
of another, and the subsequent entire broad optical OFDM superchannel signal spectrum is seamless. Consequently, with the frequency guard-bands eliminated, the SE is greatly improved which is an extremely important target for long-haul superchannel transmission systems.

Besides increasing the system SE, the use of optical frequency comb also guarantees that the phase relationship among the different spectral optical slices of OFDM-bands is preserved after coherent detection [17]. This presents an alternative means to achieve spectral overhead reduction for PA-CPR algorithm [25] through joint-carrier phase tracking. Particularly, the phase noise estimate can be shared among the multiple sub-bands where one estimated CPE is applied to demodulate all the individual different OFDM-bands. In this context, the pilot-overhead and CC associated with the popular frequency-domain PA-CPR will be ultimately scale down by a factor $K$, compared to using independent $\mathrm{K}$ pilot-subcarriers and $\mathrm{CPR}$ modules for the same purpose in conventional multiwavelength OFDM-based superchannel systems. To the best of our knowledge, this remains to be investigated especially for long-haul optical fiber transmission of multi-wavelength comb-based orthogonal band-multiplexed OFDM-based superchannel signals. In particular, joint-carrier phase tracking is recently shown to suffer performance degradation due to dispersion-induced phase-offset in comb-based Nyquist-WDM superchannel transmission systems [13], [14]. Since CO-OFDM systems with adequate cyclic prefix (CP) exhibit robust immunity against residual chromatic dispersion (CD) [40], it will be interesting to investigate the joint-carrier phase tracking for multiwavelength OFDM-based superchannel systems enabled by optical frequency combs, which is the focus of this paper.

In this paper, we have designed a master-slave joint-carrier phase tracking scheme for OFDM-based superchannel transmission system enabled by optical frequency combs. In this scheme, the phase noise of all sub-bands of the multi-band OFDM superchannel signal is compensated by using only one phase estimate from the central sub-band. Consequently, the spectral overhead and the computational complexity associated with the popular frequency-domain pilot-subcarrier common phase error estimation and compensation is significantly reduced by a factor $K$. The performance of this jointcarrier phase tracking scheme has been evaluated successfully via comprehensive simulation, where results show that the BER threshold for soft-decision FEC could be achieved for 50GHz-spaced 5band M-ary QAM (M = 4, 8, 16 \& 32) OFDM superchannel signal with zero guard band and both laser and nonlinear phase noise effects after $7000 \mathrm{~km}, 4000 \mathrm{~km}, 3000 \mathrm{~km}$ and $2000 \mathrm{~km}$ SSMF transmission respectively. The simulation results show that there exist dispersion-induced differential phase offset among OFDM-bands whose impact on joint-carrier phase tracking is dependent on the modulation format, channel count and fiber length. In this case, the differential phase offset degrades the joint-carrier phase tracking performance of outer sub-bands than the inner sub-bands. Finally, we demonstrate experimentally the feasibility performance of the designed master-slave carrier phase tracking technique for an OFDM-based superchannel enabled by optical frequency combs. 


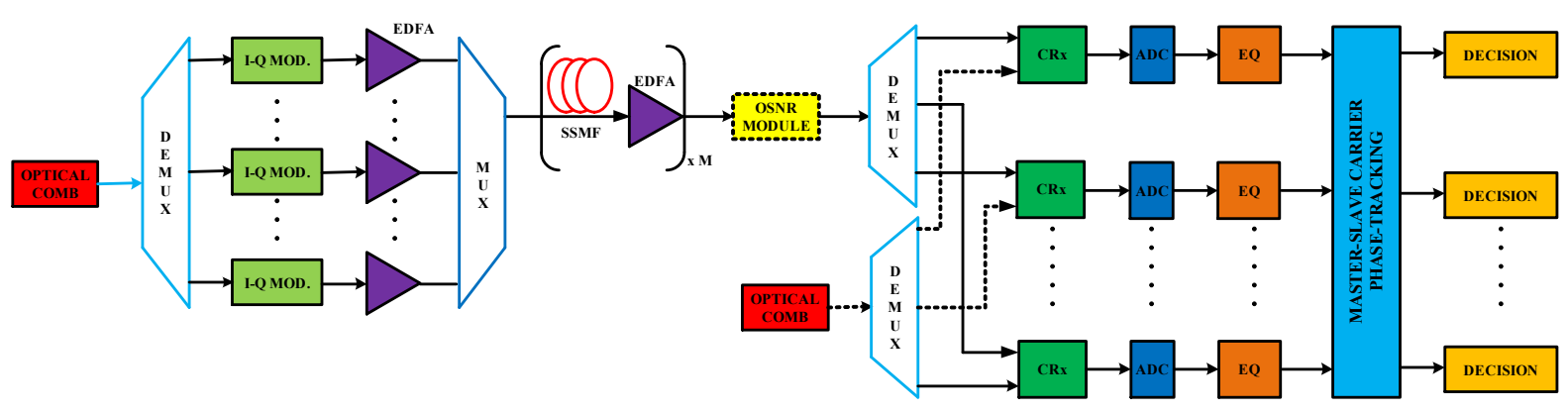

Fig. 1. Schematic for standard comb-based WDM superchannel transmission system set-up. I-Q MOD.: In-phase-Quadrature modulator; DEMUX/MUX: demultiplexer/multiplexer; CRx: coherent receiver; EQ: equalizer; ADC: analogue-to-digitalconverter; erbium-doped fiber amplifier (EDFA), SSMF: standard single-mode fiber.

\section{Principle of Proposed Master-Slave Carrier Phase Tracking Method for multi-band OFDM-BASED SUPERCHANNEL ENABLED BY OPTICAL FREQUENCY COMBS}

In this work, we consider the use of optical frequency combs for creating standard WDM transmission of OFDM-based superchannel signals, as shown in Fig. 1 [10]. For OFDM-based superchannel transmission systems with phase-locked carriers, the carrier phase noise can be assumed to be highly correlated among the OFDM-bands [17]. This shared phase evolution among the OFDMbands provides the possibility to reuse the resources needed for phase estimation for other sub-bands within the superchannel spectrum via joint-carrier phase tracking techniques. However, as shown in [13] and [14] and also depicted in this work, there may exist band-dependent phase offset, which degrade the performance of joint-carrier phase tracking schemes. The origin of this phase offset can be traced to the different group velocity delay experienced by each OFDM-band as a result of the fiber $\mathrm{CD}$, which is adequately captured by the baseband frequency domain $\mathrm{CD}$ transfer function expressed as [11]:

$$
H_{C D}(L, \omega)=\exp \left(j \frac{D \lambda^{2} L}{4 \pi c} \omega^{2}\right)
$$

where $D$ denotes the dispersion coefficient, $L$ is length of the transmission fiber link, $\lambda$ represent the carrier wavelength, $c$ is the velocity of light and $\omega$ is the corresponding frequency of the channel. It can be seen clearly from (1) that the transfer function is centered at the frequency of the corresponding channel. In this case, the fiber dispersion profile will be different for the different frequency bands so that the OFDM-band on each wavelength channel will potentially experience different channel responses during the forward propagation along the fiber. On the other hand, the carrier phase noise originates independently from the linewidths of the transmitter and local oscillator (LO) lasers. As a result of the different group velocity delay experienced by the individual OFDMbands, the bands would gradually walk off each other after fiber transmission and subsequently arrive at the receiver at different time instants and mix with different parts of the LO phase noise. This is because the carrier phase after fiber transmission will have different delays in the different channels, where the relative CD-induced time-delay between the channels can be expressed as [13], [14]: 


$$
\Delta T_{N_{1}, N_{2}}=\frac{D L R_{s} \lambda_{0}^{2}(1+\alpha)}{c K}\left|N_{1}-N_{2}\right|
$$

where $\lambda_{0}$ denotes wavelength of the central channel, $R_{s}$ is the system baudrate, $\alpha$ is the roll-off factor of the root-raised cosine (RRC) pulse-shaping filter used to confine the waveform of each channel, $K$ is the number of channels within the superchannel with $N_{1}$ and $N_{2}$ as the indices of the channels under consideration. It is clear from (2) that the relative time-delay depends on the accumulated $\mathrm{CD}$ of the fiber. Consequently, the interplay between fiber CD and LO laser linewidth will cause significant wavelength-dependent $\mathrm{CD}$-induced phase-rotation in detection, which will eventually degrade the broadband phase coherence among the channels. Particularly, if the OFDM signals on individual wavelength channels are processed independently before CPR, the CD-induced phase noise will manifest as a phase offset between the different OFDM-bands. Since joint-carrier phase tracking schemes rely on the phases being similar, the carrier phase noise cannot be perfectly removed by applying the common phase estimate across the entire superchannel.

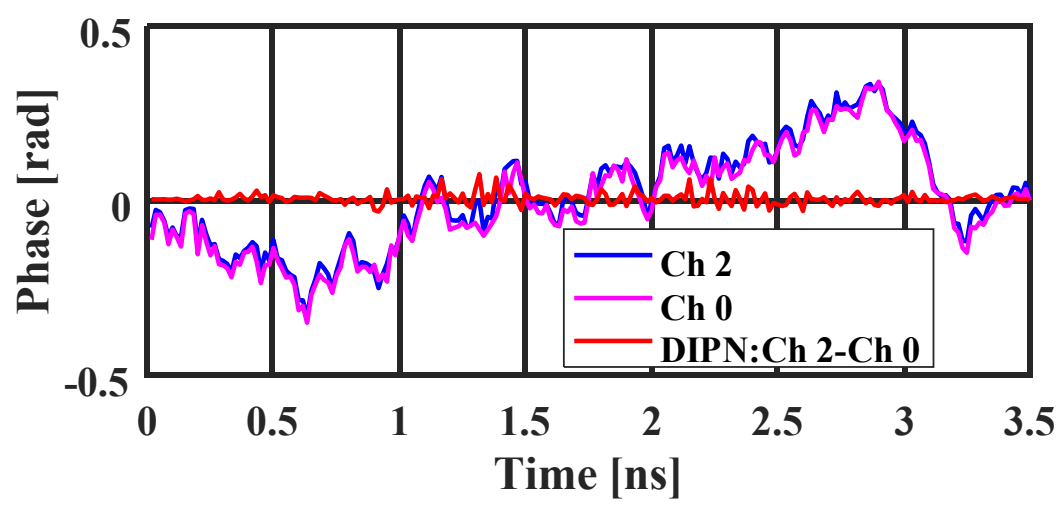

(a)

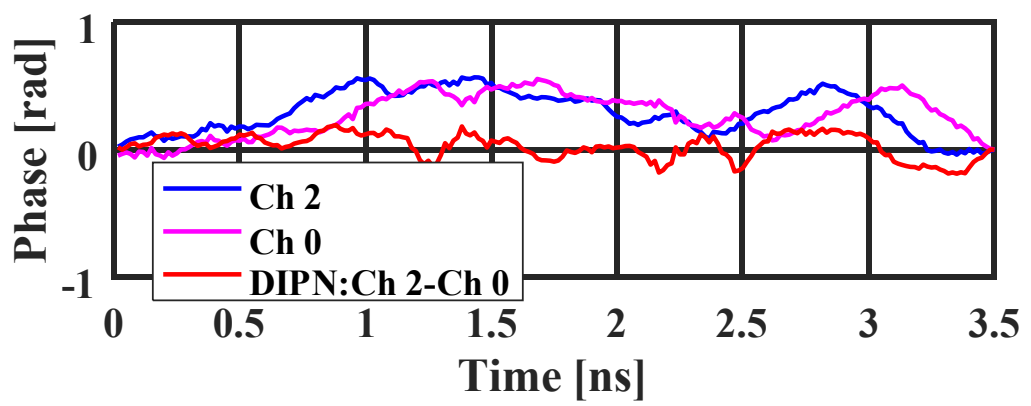

(b)

Fig. 2. Comparison of independently recovered phase fluctuations for two 4-QAM OFDM-bands on wavelength channels with indexes $\mathrm{Ch} 2$ and $\mathrm{Ch} 0$, taken out of simultaneously received 50GHz-spaced 5-band superchannel after $3000 \mathrm{~km}$ linear fiber transmission distances, when considering only (a) transmitter comb laser linewidth, and (b) LO comb laser linewidth.

For each case, the linewidth is set to $100 \mathrm{kHz}$ without ASE noise, where the recovered phases (together with the phase difference between them) are computed after the accumulated $\mathrm{CD}$ is compensated at the receiver using digital signal processing (DSP).

To confirm the origin of this CD-induced phase noise and characterize its phase variation speeds for comb-based OFDM superchannel systems, we recovered the phases of two 4QAM-OFDM-bands on wavelength channels with indexes Ch 2 and Ch 0 using independent PA-CPR [25] for each channel. The two bands are taken out of 5 simultaneously received OFDM-bands spaced at $50 \mathrm{GHz}$ after 
$3000 \mathrm{~km}$ linear fiber transmission distance. The recovered phases are plotted in Fig. 2, together with the phase difference between them, where we use the phase recovered from the central channel $\mathrm{Ch} 0$ as a reference to determine the $\mathrm{CD}$-induced phase noise. Comparing the independently extracted phase traces, it can be seen clearly from Fig. 2 (a) that the recovered phases match closely when only the laser linewidth of the transmitter comb is considered. However, when only the laser linewidth of the receiver LO comb is considered, small discrepancies can be observed in the recovered phases. It is obvious that the fiber CD induces noticeable phase difference between all OFDM subcarriers which may decorrelates the common phase error between them. In this case, the phase noise imparted to each OFDM-band by the LO comb laser linewidth may be determined by the CD-induced walk-off effect. This confirms our hypothesis that the interaction between the fiber CD-induced walk-off effect among the OFDM-bands and LO laser linewidth give rise to intra-band CD-induced differential phase noise, which will degrade performance of joint-carrier phase tracking techniques. For convenience here, we adopt the name "dispersion-induced phase noise (DIPN)" or "differential phase offset" to distinguish this unique phenomenon from the original common phase error among the subcarriers across the different OFDM bands. Also, it can be observed from Fig. 2 that the differential phase offset is typically much smaller and drifts in orders of magnitude much slower than the common phase noise derived directly from the linewidths of the carrier and LO comb laser sources. For this reason, the similarity among phase traces or phase fluctuations of the different OFDM-bands is enough to enable joint-carrier phase tracking in this work.

For different objectives and benefits, there are generally two main strategies for implementing jointcarrier phase tracking in comb-based superchannel systems: master-slave (MS) and what can be described as multi-channel averaging (MCA) joint-carrier phase tracking schemes. As the name implies, the MS-CPR reuse the phase estimated from one (i.e., the master channel) for the other channels (i.e., the slave channels), which reduces the CC of the CPR unit. For MCA-CPR, the carrier phase is estimated using two or more (or all) channels, where the additional phase information is used to improve the phase-tracking speed (i.e., phase-noise tolerance) in phase noise limited systems, while maintaining the same tolerance to additive noise. In this context, the system understudy dictates the optimal design for joint-carrier phase tracking scheme, especially as the impact of fiber transmission effects on CO-OFDM is system-specific. Therefore, as shown in Fig. 3, we designed a MS-CPR scheme that is capable of compensating both the common phase noise as well as the differential phase offset in OFDM-based superchannel transmission systems enabled by optical frequency combs. In the designed master-slave CPR scheme, we use the pilot-symbols allocated on few dedicated pilotsubcarriers across the OFDM-band on the central wavelength channel (i.e., channel Ch 0 in Fig. 1) to estimate the common phase error, which is applied to correct the phase drift of all subcarriers across the different sub-bands within the OFDM-based superchannel. This is similar to the master-slave carrier phase tracking scheme previously demonstrated for comb-based spectral [13], [14], [41] and spatial [6] superchannel transmission systems as well as the multi-band DFT-spread OFDM system in 
[42]. Since the pilot-subcarriers used occupies a small fraction of the overall OFDM bandwidth, this MS-CPR scheme will ultimately serve as alternative CPR method to achieve reduction in overall pilot-subcarrier overhead plus CC in CO-OFDM systems.

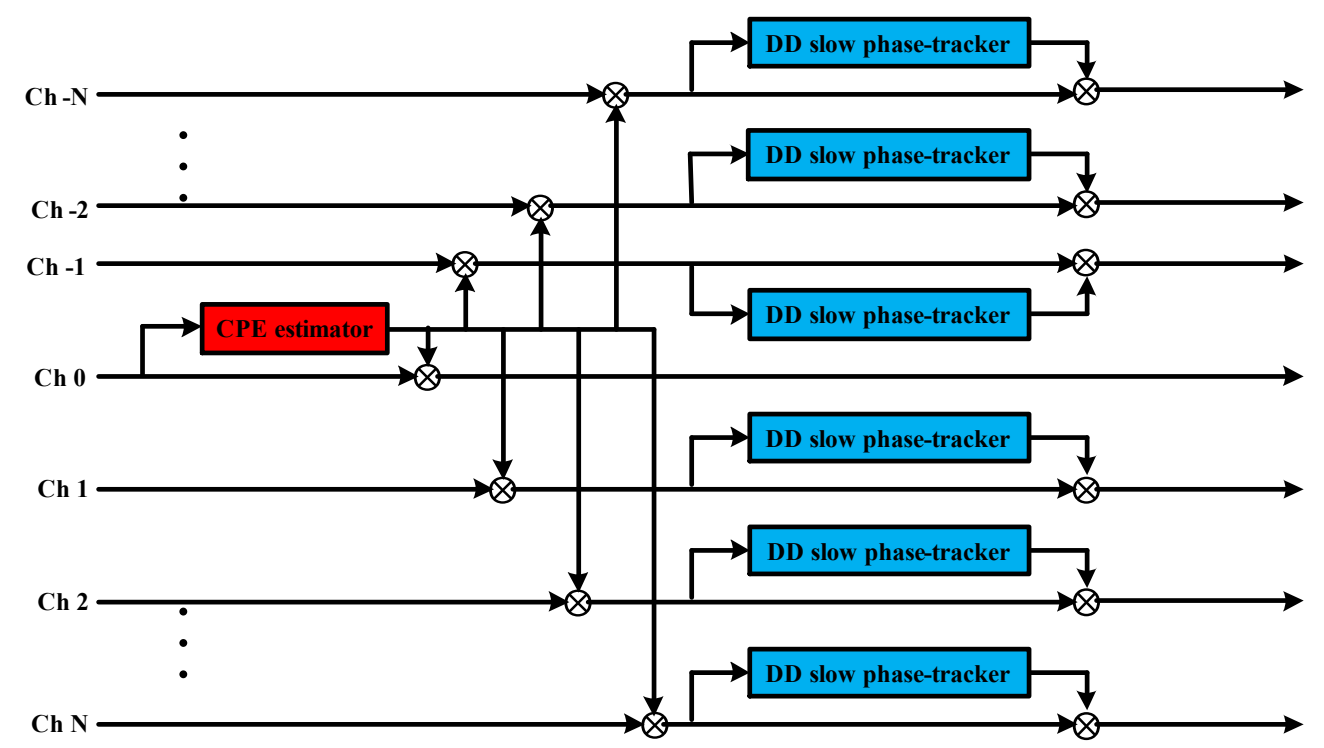

Fig. 3. Schematics of designed master-slave joint-carrier phase-tacking scheme for comb-based WDM superchannel transmission system. CPE: common phase error; DD: decision-directed; N: channel index.

Due to the differential phase offset, the phase noise in the slave OFDM-bands cannot be perfectly removed by applying the master common phase estimate, which is only synchronized with the OFDM-band on the central wavelength channel. Thus, the differential phase offset will remain on the slave OFDM-bands after the master phase estimate has been applied to them, which will degrade system performance. Therefore, after master CPE compensation, the signals on the slave OFDMbands are fed into slow phase-trackers to compensate any remaining differential phase offset on them. In this work, the slow phase-tracker is implemented as a single-tap decision-directed (DD) adaptive equalizer. As the tracking-speed requirements of the differential phase offset are previously shown to be low, the slow DD phase-tracker used should suffice to remove any small differential phase offset remaining on the slave OFDM-bands after master phase compensation. Advantageously, this phasetracker functionality can potentially be integrated into some existing adaptive equalizers that performs polarization demultiplexing prior to CPR, e.g. DD least mean square (DD-LMS) [43]-[45], differential phase compensated constant-modulus [46] algorithms, provided that the error signal used for the tap update is sensitive to phase rotations. In this context, the complexity added by this phasetracker is small compared to using a separate CPR block for each OFDM sub-band that would have been required in the absence of master-slave joint-carrier phase tracking. Consequently, the differential phase offset tracking scheme is computationally lightweight compared to the master phase estimator, which is the goal of any master-slave CPR scheme.

To verify our designed MS-CPR scheme, in Fig. 4(a), we compare the BER performance as a function of OSNR for the MS-CPR with (w) and without (w/o) the DD slow phase-tracker. It can be seen clearly from Fig. 4(a) that the designed MS-CPR scheme is obviously capable of compensating 
the carrier phase noise in OFDM-based superchannel systems with phase-locked carriers. From the central channel point of view, the results depicted in Fig. 4(a) show that the differential phase offset can easily be tracked with a fine phase estimator, and hence does not affect system performance when the CPR is performed independently for each OFDM-band. For this reason, in Fig. 4(b), we use the independent CPR (I-CPR) performance as a baseline to compare and assess the performance of our proposed MS-CPR scheme. As can be seen clearly from Fig. 4(b), the result confirmed that the performance of our master-slave phase tracking scheme closely follows the performance of the conventional I-CPR method, at least for the systems studied here.

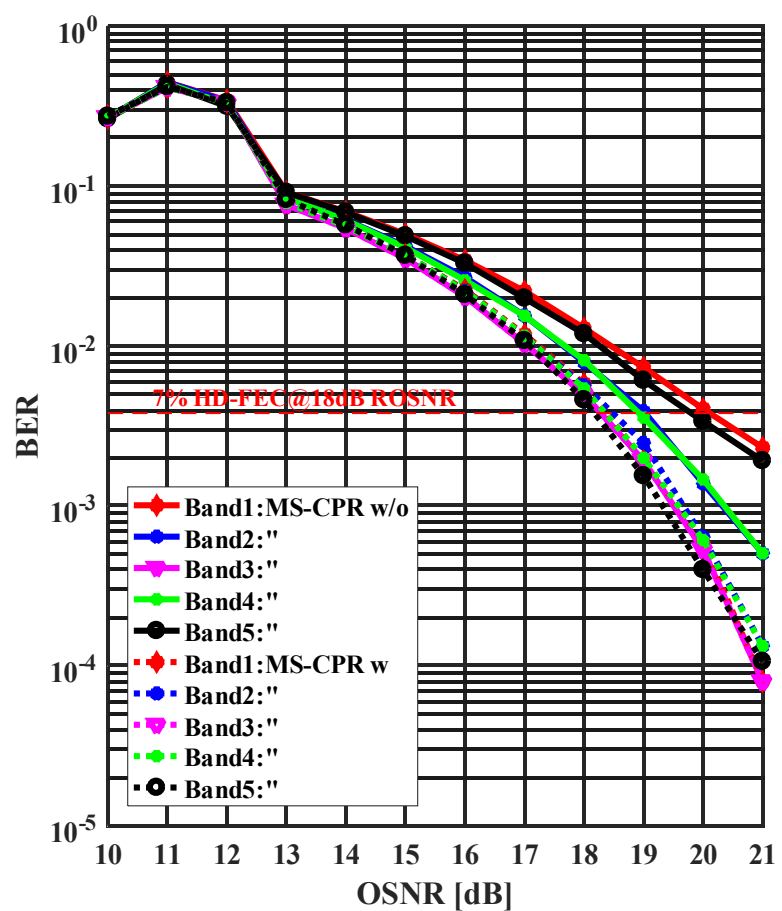

(a)

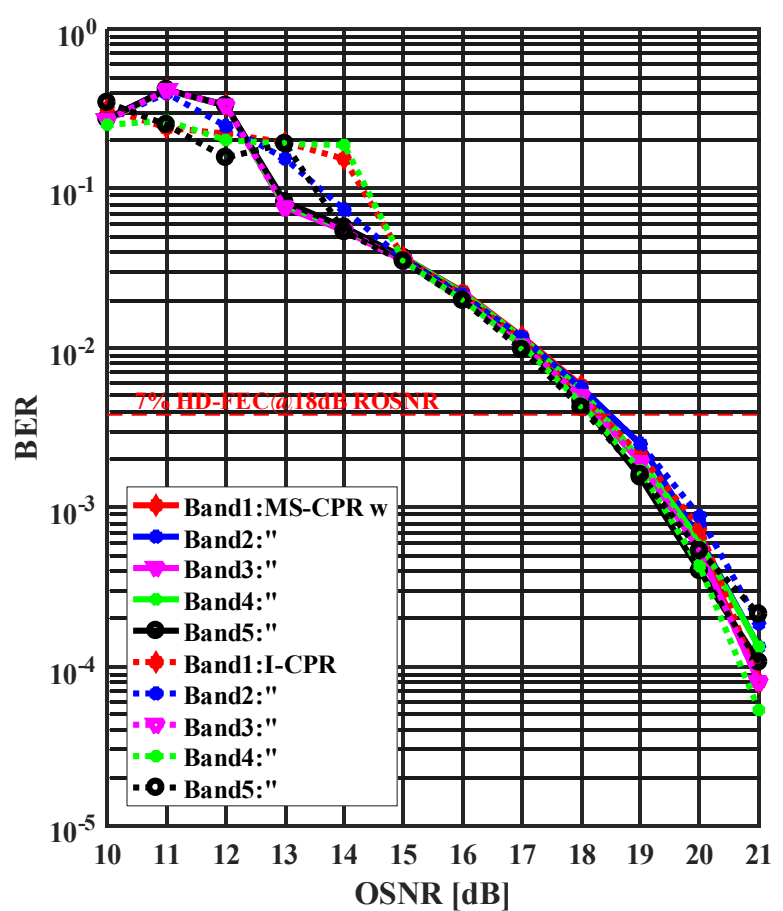

(b)

Fig. 4. BER as a function of OSNR for (a) MS-CPR with (w) and without (w/o) DD slow phase-tracker, (b) MS-CPR with

DD slow phase-tracker (MS-CPR w) versus I-CPR, after 4000km linear fiber transmission of 50GHz-spaced 5-channel 4QAM OFDM-based superchannel: The linewidth was set to $200 \mathrm{kHz}$, accounting for both signal and LO laser linewidths. ROSNR: required OSNR.

\section{NumERICAL SimUlations FOR PERFORMANCE EVALUATION}

In this section, based on the set-up shown in Fig. 1, we carry out simulation of OFDM-based spectral superchannel transmission system with phase-locked carriers to evaluate the performance of the designed master-slave joint-carrier phase tracking scheme shown in Fig. 2. All numerical simulations were performed with VPItransmissionMaker v9.1 integrated with MATLAB R2016b for transceiver DSP. The superchannel had five WDM channels spaced at $50 \mathrm{GHz}$ with zero guard band, and modulated using OFDM based on four different modulation formats: 4-QAM, 8-QAM, 16-QAM and 32-QAM. The nominal baudrate is 50 Gbaud per channel which translate into nominal bit-rate of $100 \mathrm{~Gb} / \mathrm{s}, 150 \mathrm{~Gb} / \mathrm{s}, 200 \mathrm{~Gb} / \mathrm{s}$ and $250 \mathrm{~Gb} / \mathrm{s}$ for 4-QAM, 8-QAM, 16-QAM and 32-QAM, respectively. The optical comb was generated using 5 independent carrier and LO lasers spread around the frequency $193.1 \mathrm{THz}$, which serves as the central channel. For each modulation format, the 
5 carriers were respectively injected to multiple parallel I-Q modulators which are driven by different pseudo-random bit sequences (PRBS) to generate the respective transmitted symbols in order to emulate independent data transmission in each OFDM-band. Among the different flavours of OFDM techniques demonstrated in literature [40], [47], the conventional FFT-based OFDM [16], [17] is employed for this study. Table I lists out the typical parameters used in this work. The guard interval for each OFDM-band was kept smaller because the fiber CD was compensated by the receiver DSP. Since the commonly used pilot-aided estimation technique [25] is applied for the common phase error estimation for all the four modulation formats in this work, 10 pilot subcarriers out of the 90 data subcarriers on the central OFDM-band was dedicated for the common phase error estimation and compensation across the entire OFDM-based superchannel.

\begin{tabular}{cc} 
TABLE I. LIST OF TYPICAL PARAMETERS USED \\
\hline Parameters & Values \\
\hline (I)FFT size & 128 \\
Data subcarriers & 90 \\
Guard interval & 12 \\
SSMF span length & $100 \mathrm{~km}$ \\
Attenuation & $0.2 \mathrm{~dB} / \mathrm{km}$ \\
Dispersion & $16 \mathrm{ps} / \mathrm{nm} / \mathrm{km}$ \\
Nonlinearity & $2.6 \times 10^{-20} \mathrm{~m}^{2} / \mathrm{W}$ \\
EDFA Noise figure & $5 \mathrm{~dB}$ \\
Total Laser Linewidth & $200 \mathrm{kHz}$ \\
\hline
\end{tabular}

After modulation, all the OFDM-bands are wavelength-multiplexed in the optical domain and then launched into an EDFA-amplified fiber link with the launch power per channel controlled by a booster EDFA. In order to ensure sufficient OSNR for signal quality evaluation, the fiber transmission distance was adapted to the different modulation formats: 70x100 km for 4-QAM, 40x100 km for 8QAM, 30x100 km for 16-QAM and 20x100 km for 32-QAM signals. The in-line EDFAs is modelled with a gain of $20 \mathrm{~dB}$ to equalize the fiber loss. In order to control the OSNR and measure the BER during linear fiber transmissions, an AWGN noise loading device (i.e., an OSNR module) is used at the input of the receiver. For nonlinear fiber transmissions, the OSNR module is removed and ASE noise is loaded using the noise figure of the in-line EDFA to emulate the nonlinear phase noise (NLPN) in practical systems. After fiber transmission, each OFDM-band is coherently detected using a standard coherent optical receiver which is same for all the four modulation formats. As joint-carrier phase tracking schemes require preservation of the relative phases between OFDM-bands at both the transmitter and receiver, phase-locking of the lasers is achieved by setting the random number seed (RNS) parameter to same non-zero value, but the RNS value used for the carriers is different from the LOs.

Following analogue-to-digital conversion, a linear compensator (LC) is applied per OFDM-band to compensate only CD effects in the frequency domain [48]. Otherwise, a nonlinear compensator (NLC) 
is implemented per sub-band where the signal is digitally back-propagated (DBP) to simultaneously mitigate fiber nonlinearities and compensate CD effects [49] Multi-channel DBP is not employed here since imperfect phase synchronization between the LOs may give rise to further phase error and cause degradation of its performance [16]. Since frequency offset (FO) will be similar for all the OFDMbands and can easily be compensated using master-slave FO compensation technique, frequency synchronization issues were not considered in this work in order to focus on phase noise effects. Next, we applied the MS-CPR scheme designed in Fig. 2 to remove the phase fluctuations of the superchannel. Firstly, we applied the pilot-aided master phase estimate obtained from the 10 pilotsubcarriers allocated across the central OFDM-band (i.e. the master band) to compensate the common phase error of all subcarriers across the different OFDM-bands for all the four modulation formats. After the master-slave CPE compensation, the signal on each slave OFDM-band is fed into a slow DD phase-tracker to compensate any differential phase offset remaining on them. Finally, the output data stream for each OFDM-band is sent to the decision device for BER calculation.

\section{Performance Evaluation Results of Proposed Master-Slave Joint-Carrier Phase RECOVERY METHOD}

In this section, we focus on the performance of the master-slave joint-carrier phase tracking method designed in Fig. 2 for coherent optical transmission of OFDM-based superchannel system with phaselocked carriers based on four different M-ary QAM $(\mathrm{M}=4,8,16$ and 32) formats with laser and nonlinear phase noise effects. Firstly, we investigated the performance of the proposed MS-CPR scheme with or without the slave slow DD phase-tracker for linear fiber transmission with laser linewidth effects only. The results of this investigation are depicted in Fig. 5 for all the four modulation formats, which once again the capability of our proposed CPR technique in compensating the carrier phase noise in comb-based OFDM-WDM superchannel transmission systems. For same data rate, it can be observed from Fig. 5 that lower-order M-QAM formats would exhibit a better tolerance to the differential phase offset, even though they suffer more $\mathrm{CD}$. This is because the higher-order modulation formats with shorter fiber transmission distance suffers stronger effects of differential phase offset, compared to the lower-order modulation formats, indicating that sensitivity of the differential phase offset increases with the order of the modulation formats. Similar to the comb-based Nyquist-WDM superchannel systems [13], [14], it can be observed from Fig. 5 that performance of MS-CPR without the slave DD phase-tracker for the outer OFDM-bands is adversely impacted more by the differential phase offset than the inner OFDM-bands. Thus, the side OFDMbands suffer more significant differential phase offset and exhibit worse performance compared to the inner OFDM-bands. The reason for this performance difference can be attributed to the fact that optical frquency combs are characterized by two basic frequencies: the central frequency and the comb line spacing. 


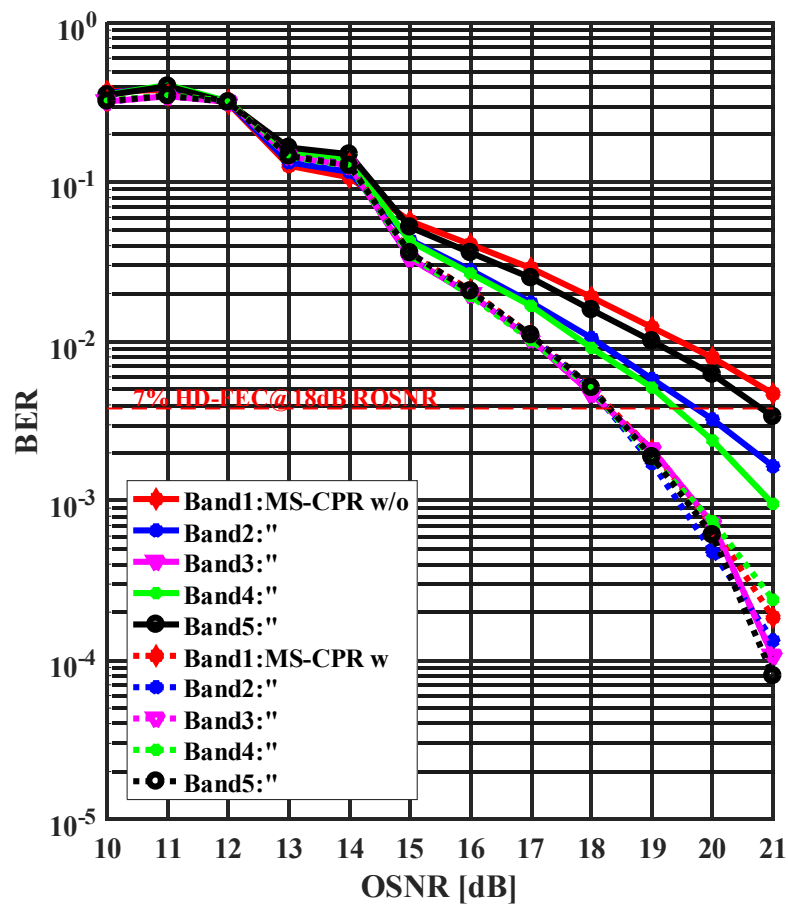

(a)

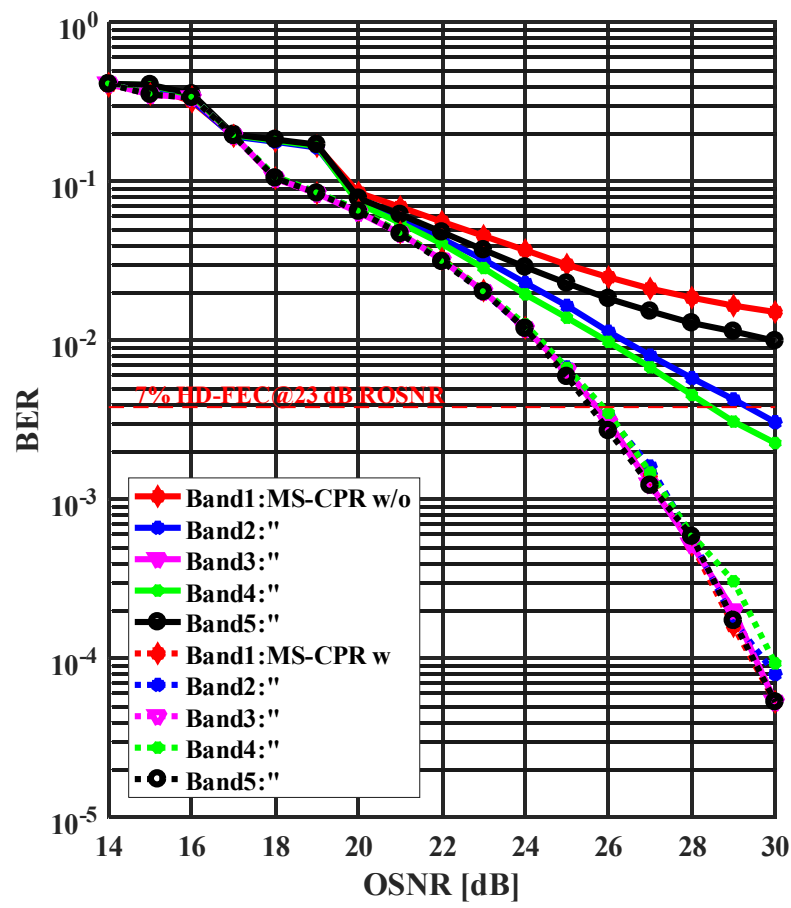

(c)

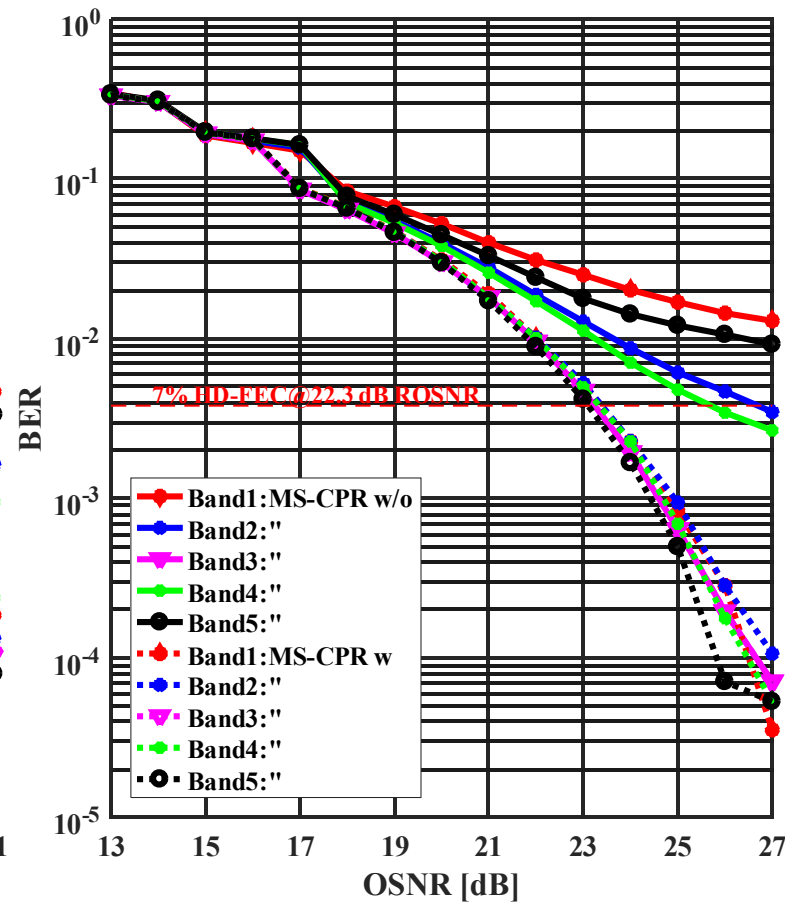

(b)

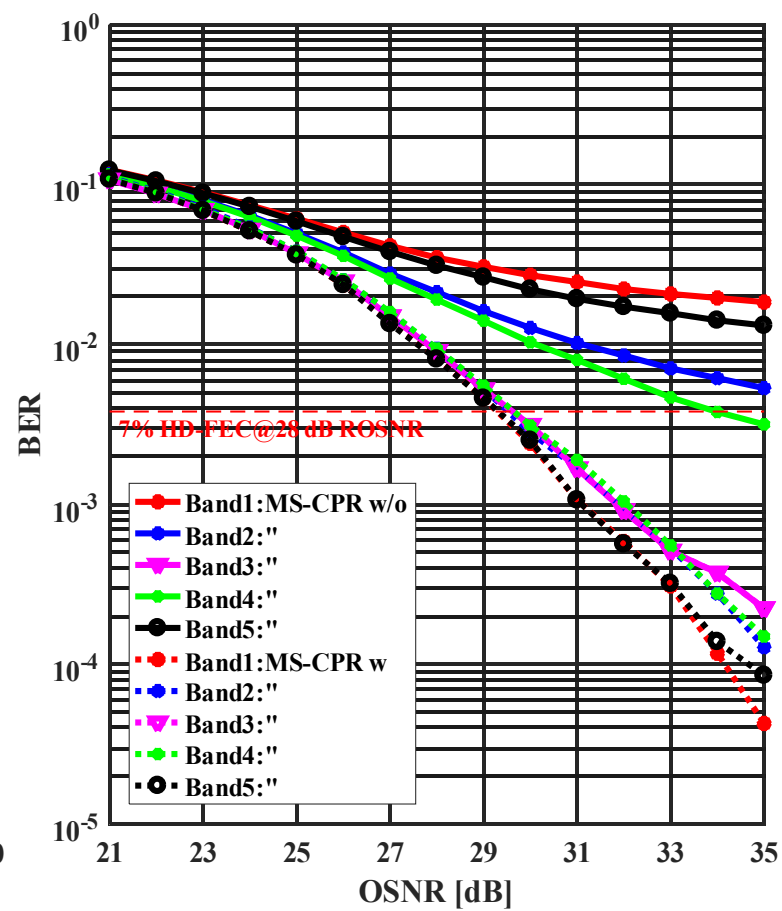

(d)

Fig. 5. BER performance as a function of OSNR for linear fiber transmission for the four different modulation formats: (a) 4-QAM, (b) 8-QAM, (c) 16-QAM and (d) 32-QAM using the MS-CPR with (w) and without (w/o) the slave slow DD phase-tracker.

In effect, any phase-difference between the comb lines will scale with frequency spacing between the central and side frequencies, and fiber length (i.e., accumulated CD). This is in agreement with phase of the baseband frequency domain CD transfer function in (1), where the phase can be seen to vary quadratically with the corresponding frequency. In this case, the phase will increase its slope at a greater frequency separation from the central frequency [11]. Thus, for same perturbation in the 
carrier frequency caused by a frequency shift due to the LO laser linewidth, the phase variation caused by the perturbation at a greater frequency separation from the central frequency will be larger than the phase variation induced by the frequency perturbation at a lower frequency separation. Consequently, for all the four modulation formats, the impact of differential phase offset depends on the spectral distance (i.e., frequency separation) between the side OFDM-bands and the central OFDM-band. In effect, besides the fiber length, the differential phase offset will be influenced by the channel count within the superchannel, as the number of transmitted OFDM-bands will unavoidably affect the frequency separation between the side OFDM-bands and the central OFDM-band. That is, effects of the differential phase offset is sensitive to the number of transmitted subchannels, the greater the number of sub-bands the greater the severity of differential phase offset on the side sub-bands, at least for the four modulation formats and system parameters studied in this work.

Furthermore, as nonlinear phase noise effects are unavoidable in practical fiber systems and may cause additional phase perturbations on the sub-bands, it is prudent to study the tolerance of the designed MS-CPR scheme to fiber nonlinearities, which becomes a source of performance degradation for higher-order modulation formats in long-haul fiber transmission systems. For this reason, next, we evaluated the MS-CPR scheme performance under practical long-haul transmission scenario where both laser and nonlinear phase noise effects are present. The BER values obtained as a function of fiber launch power for all the four different modulation formats using the MS-CPR scheme in the nonlinear regime is depicted in Figs. 6 and 7 for linear and nonlinear compensators (i.e., LC and NLC) respectively. Here, the transmission distances studied remain as in the linear case. Fig. 6 shows that when only LC is employed for nonlinear mitigation of all the four modulation formats, the optimum launch power can approximately be extended from $+1.0 \mathrm{dBm}$ to $+2.0 \mathrm{dBm}$ at a $2 \times 10^{-2}$ BER for the outer 4-QAM OFDM-bands using the MS-CPR with the slave phase-tracker, compared to the case without the phase-tracker. On the other hand, only $0 \mathrm{dBm}$ optimum launch power at a $2 \times 10^{-2}$ BER was achieved for the 8-QAM OFDM sub-bands using the MS-CPR with the slave phasetracker, whilst 16-QAM and 32-QAM OFDM sub-bands failed to achieve any meaningful BER performance. However, Fig. 7 shows that when DBP is employed, $+4.5 \mathrm{dBm},+3.0 \mathrm{dBm}$ and $+2.0 \mathrm{dBm}$ optimal launch powers can be achieved at the BER limit for soft-decision FEC for the 4-QAM, 8QAM and 16-QAM outer OFDM sub-bands respectively using the MS-CPR with the slave phasetracker. This is a profound improvement in the optimal launch powers of about $3.5 \mathrm{dBm},+1.0 \mathrm{dBm}$ and $+2.0 \mathrm{dBm}$ for the 4-QAM, 8-QAM and 16-QAM OFDM sub-bands respectively. Even with DBP, the 32-QAM OFDM-band again failed to achieve the BER threshold for soft-decision FEC. Above all, for all the four modulation formats, it can be seen from both Figs. 6 and 7 that the inner OFDM subbands exhibits a slightly worse performance than the outer sub-bands in both cases of LC and DBP. The reason for this poor performance may be that the inner sub-bands experience more significant nonlinear interference than the outer OFDM-bands due to inter-channel nonlinearities. 


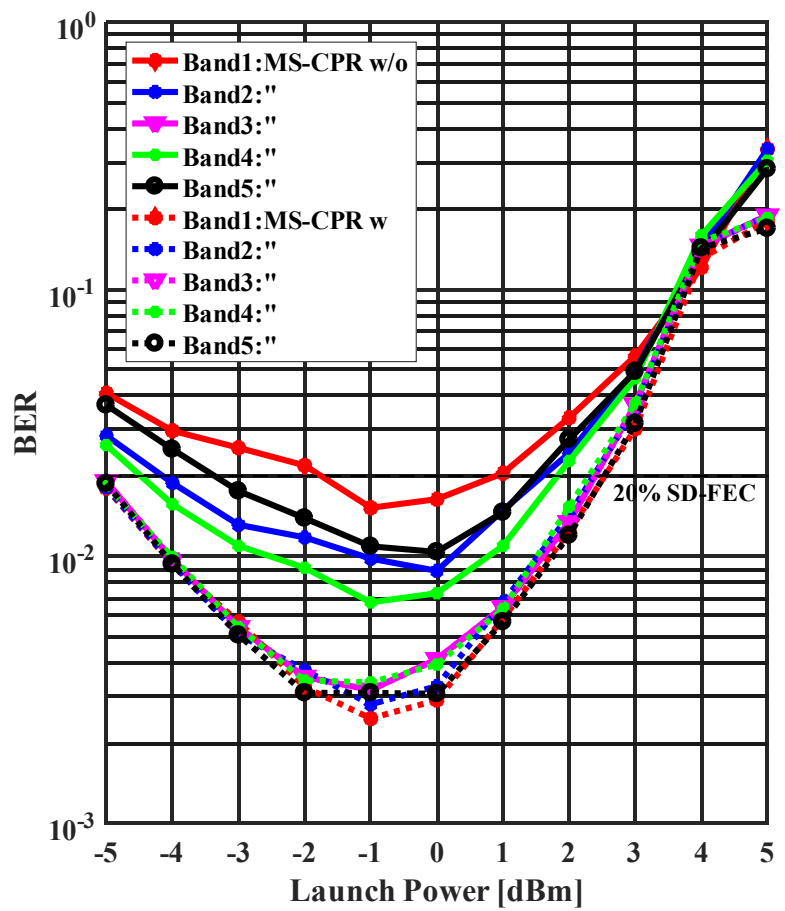

(a)

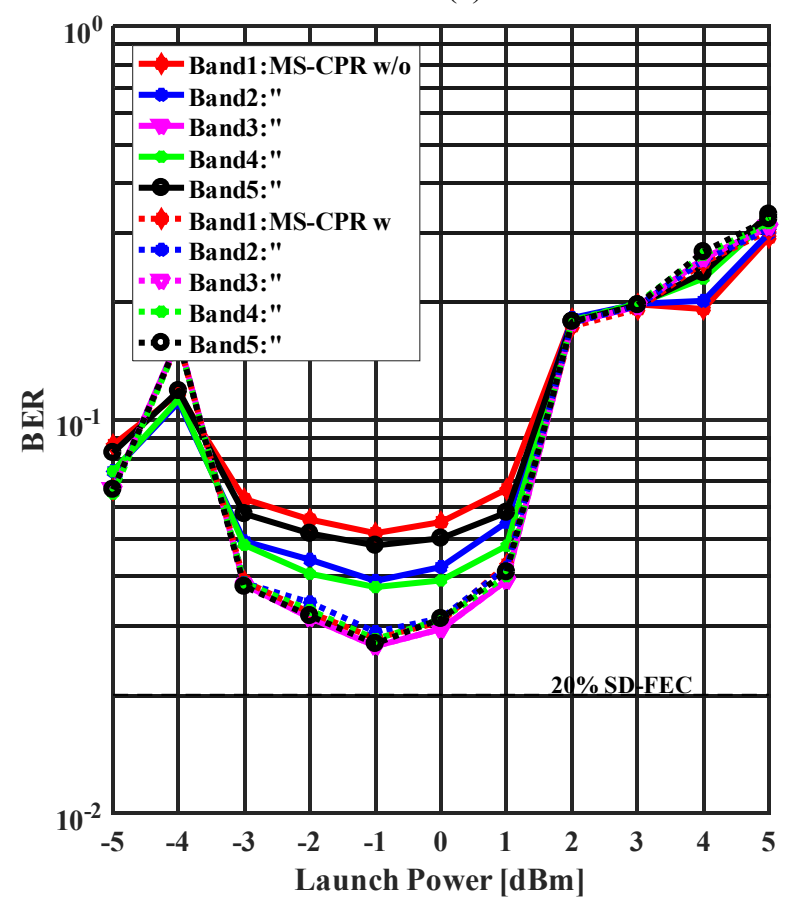

(c)

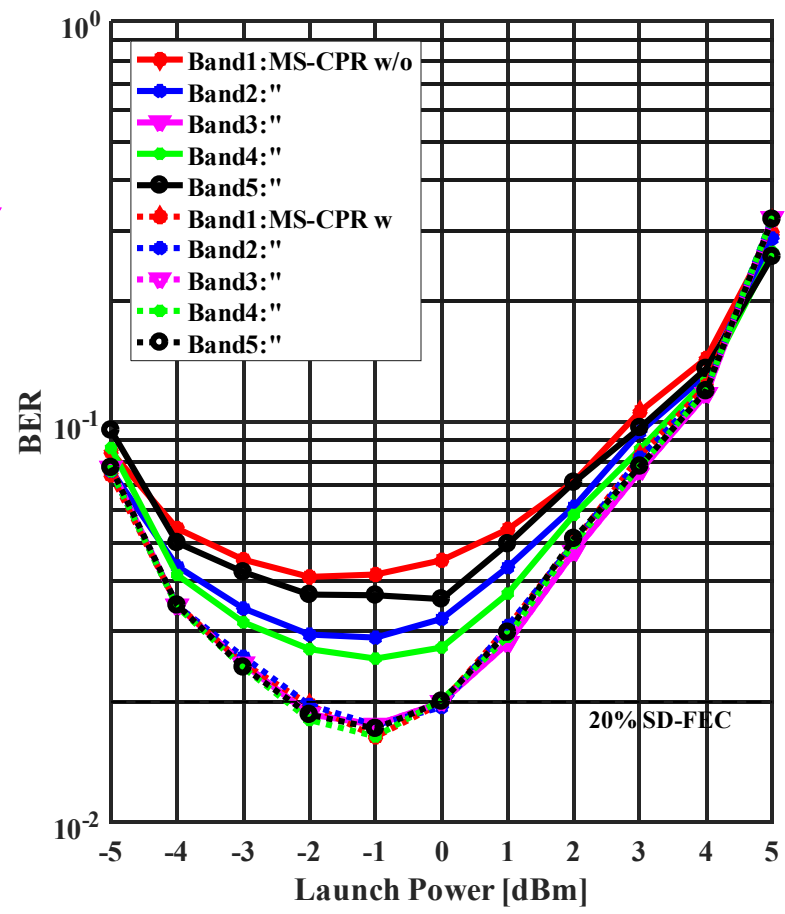

(b)

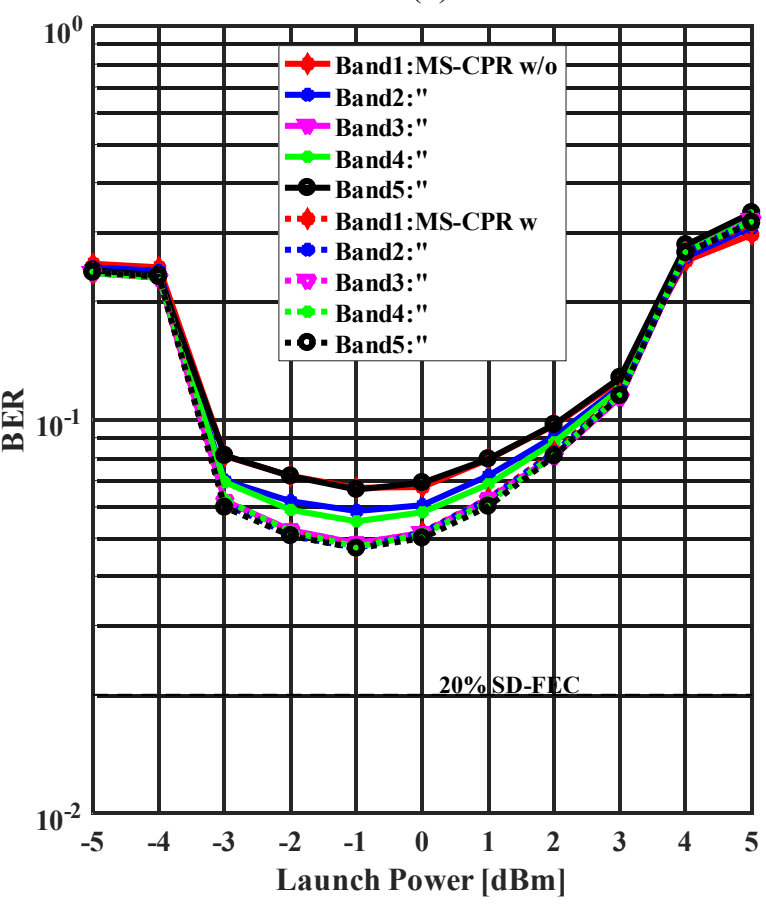

(d)

Fig. 6. BER performance as a function of fiber launch power for nonlinear fiber transmission for the four different modulation formats: (a) 4-QAM, (b) 8-QAM, (c) 16-QAM and (d) 32-QAM using the MS-CPR with (w) and without (w/o) the slave slow DD phase-tracker after LC only. 


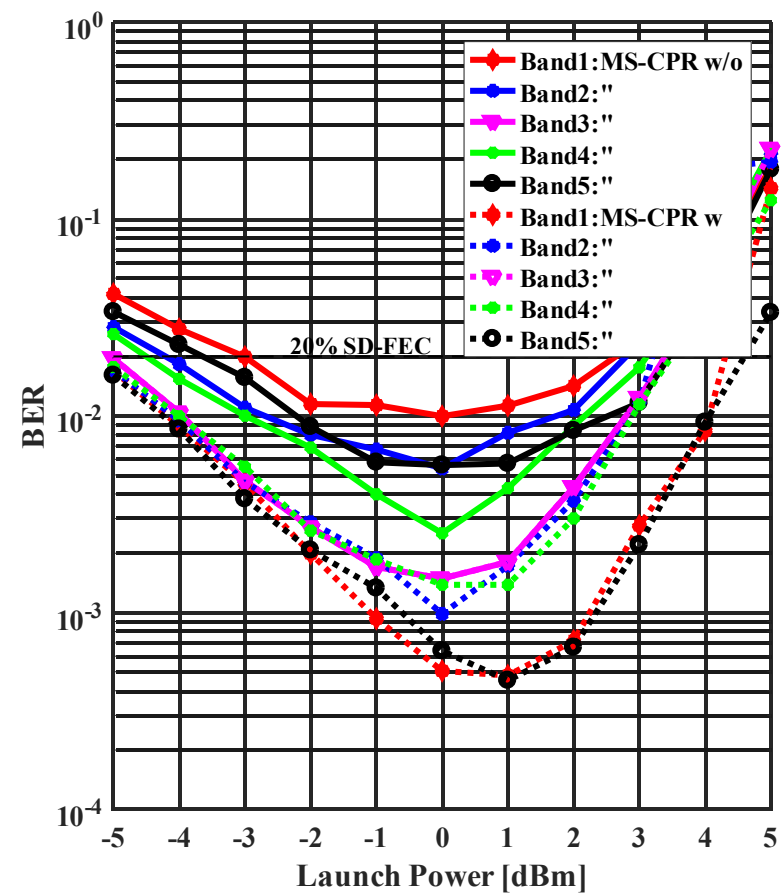

(a)

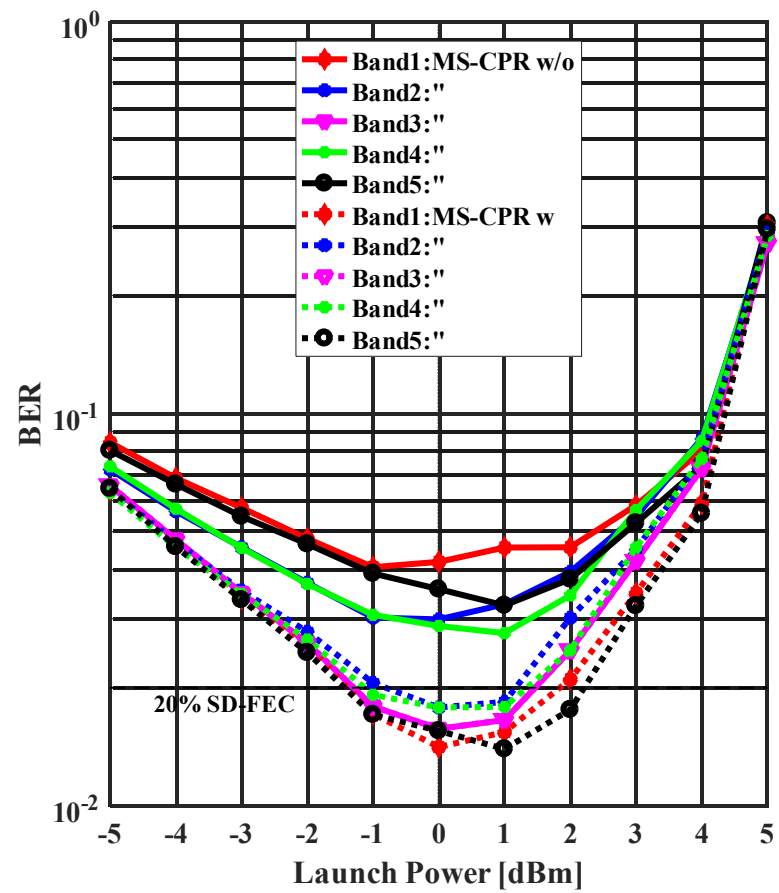

(c)

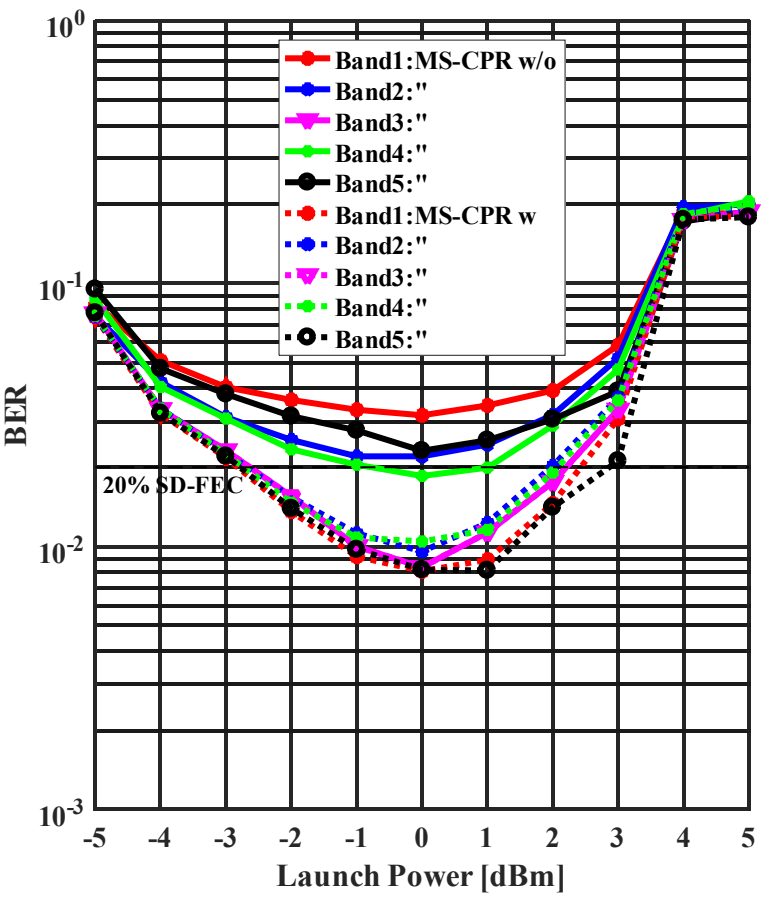

(b)

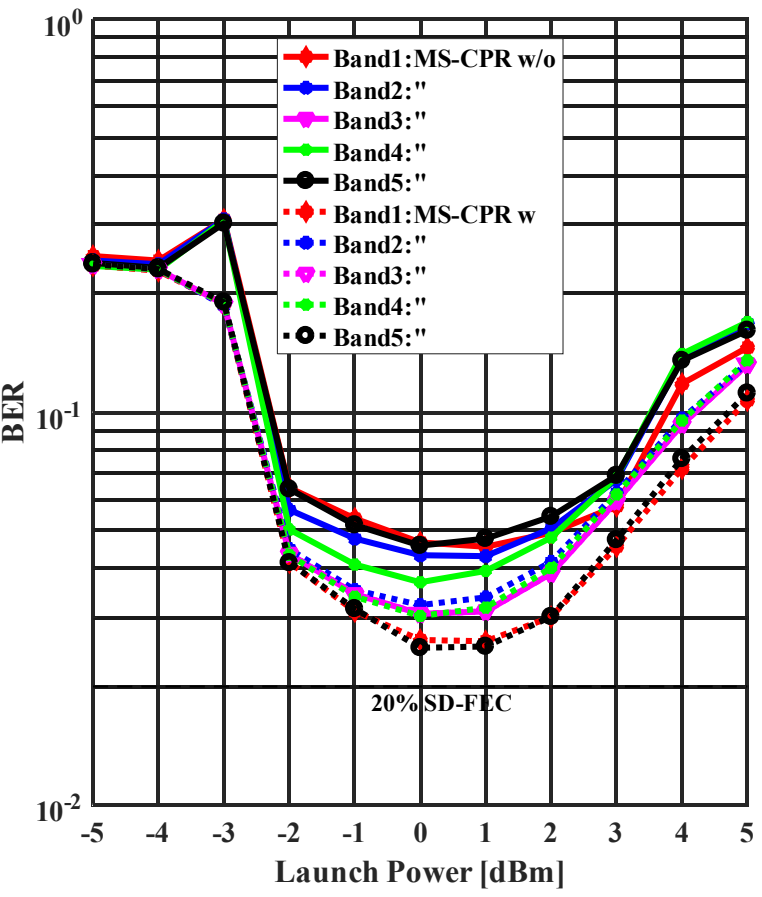

(d)

Fig. 7. BER performance as a function of fiber launch power for nonlinear fiber transmission for the four different modulation formats: (a) 4-QAM, (b) 8-QAM, (c) 16-QAM and (d) 32-QAM using the MS-CPR with (w) and without (w/o) the slave slow DD phase-tracker after DBP.

\section{Experimental Verification of Proposed Master-SlaVe CARRIER PHASE TRACKING Scheme}

Having investigated the proposed master-slave joint-carrier phase tracking technique using simulations in Section 4, in this section, we conducted two proof-of-principle experiments to demonstrate its feasibility as well as verify the phase coherence in practical OFDM-based superchannel systems enabled by optical frequency combs. Fig. 8 show schematic of the 
experimental set-up used for joint transmission and reception of comb-based WDM channels with OFDM-4QAM signals. In this work, each comb generator consists of one intensity modulator and a radio frequency (RF) oscillator source, and there are three EDFAs in the transmitter to compensate for the loss of the comb generator, the optical filters, and the optical IQ modulator. At the transmitter, applying the electro-optic (EO) modulation technique, the output light of a single continuous wave (CW) laser at $1550.4 \mathrm{~nm}$ wavelength is modulated by the intensity modulator. The intensity modulator is driven by a $0 \mathrm{dBm} 40$ $\mathrm{GHz}$ sinusoidal RF signal to generate 3 frequency comb lines spaced at $40 \mathrm{GHz}$. After amplification using EDFA, due to hardware constraints, the EO comb produced is fed through WSS to equalize the power between the lines and select only two lines for transmission. The two comb lines are amplified again and then modulated with 16-QAM random data using a single I-Q modulator. The baudrate is kept at 12.5 Gbaud, which correspond to $50 \mathrm{~Gb} / \mathrm{s}$. The time-domain OFDM data is first generated using a MATLAB program with parameters as used for the simulation. Specifically, 10 subcarriers out of the 90 data subcarriers on the central OFDM sub-band are filled with pilot-symbols which are used for the common phase error estimation across the entire superchannel.

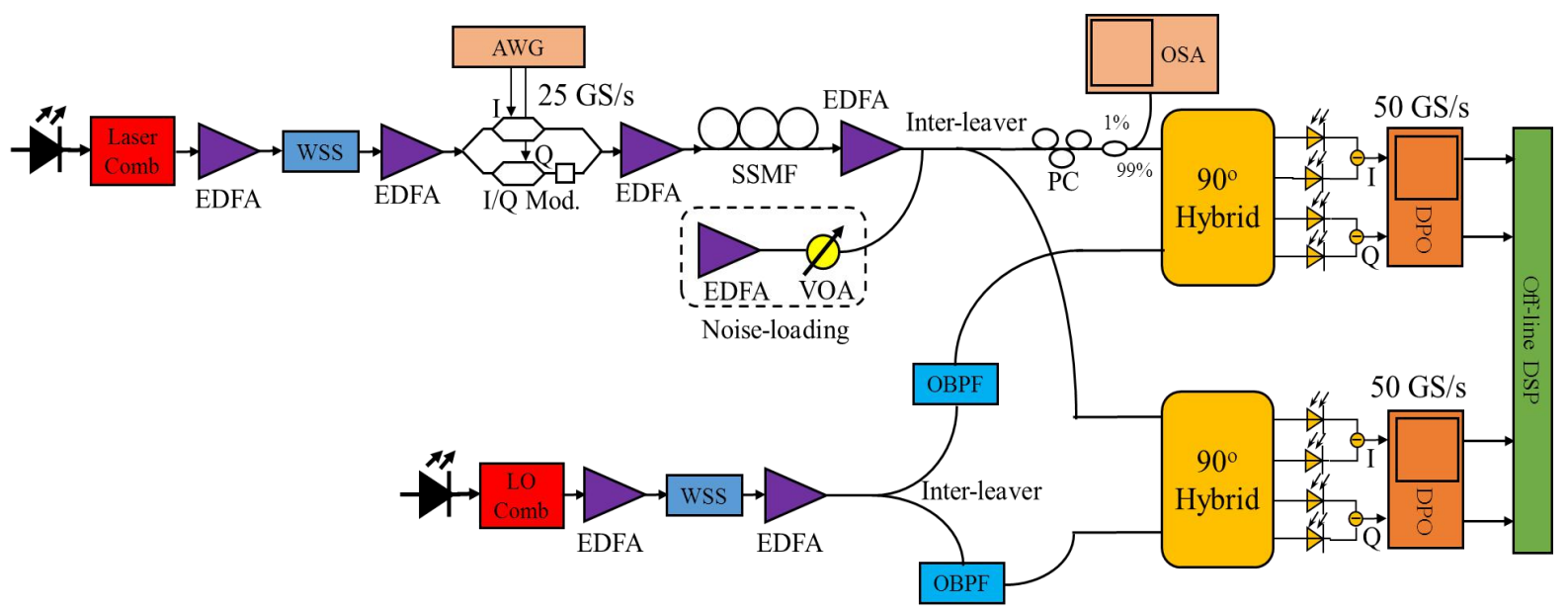

Fig. 8. Schematic of the experimental set-up for comb-based WDM transmission system: I/Q Mod.: in-phase/quadrature modulator; AWG: arbitrary waveform generator; VOA: variable optical attenuator; wavelength selective switch (WSS); OBPF: optical band-pass filter; erbium-doped fiber amplifier (EDFA).

After optical modulation, the comb signal is amplified again and sent directly to the receiver in case of back-to-back transmission. Otherwise, the comb signal is launched into a fiber transmission link, which had either one or few spans of $50 \mathrm{~km} \mathrm{SSMF}$ with typical parameters as used in the simulation. For performance evaluation, as shown in Fig. 8, a noise-loading device is used to add ASE noise to the comb signal. After transmission, the two channels are separated using a $40 \mathrm{GHz}$ interleaver and the signals on the two channels are mixed with the output of the LO comb using two synchronized standard independent 
coherent receivers. The LO frequency comb is generated in a similar manner as the transmitter comb carrier lines using a second free-running independent laser source. Specifically, the LO comb lines are selected with a second WSS and split via another $40 \mathrm{GHz}$ interleaver. In order to ensure sufficient extinction ratio, each LO line is filtered using 0.2 nm OBPF. Throughout the experiment, the laser sources used for the signal carrier and LO comb lines are specified to have a $100 \mathrm{kHz}$ linewidth to emulate external cavity lasers. The outputs from the coherent receivers are each sampled using a pair of DPO (i.e., real-time oscilloscopes) which are synchronized with a common clock signal. The sampling rate and bandwidth of the DPO are $50 \mathrm{GS} / \mathrm{s}$ and $25 \mathrm{GHz}$ respectively.

In order to ensure optimal signal in the fiber, data is captured using many launch powers. The captured data are then processed offline using a desktop PC by applying a series of the standard DSP algorithms. Specifically, optical front-end correction is first performed and the signal is resampled to keep 2 samples per symbol. Next, channel estimation and compensation is performed. After frequency offset compensation, we applied the proposed master-slave carrier phase tracking or an independent carrier phase recovery technique, both based on the pilot-aided carrier phase estimation technique, to estimate and compensate the carrier phase noise originating from the laser linewidths as well as fiber nonlinearities. Finally, BER values are obtained by comparing the bit sequence decoded from the received symbols with the original transmitted one.

Since performance of joint-carrier phase tracking schemes depends on the degree of phase coherence among the comb lines, we first study the phase noise characteristics of the electrooptic comb lines generated previously in this work. In this case, the phase coherence can better be assessed qualitatively by applying the carrier phase estimation algorithm separately to each channel and comparing their recovered phases. For this reason, in Fig. 9, we plot the independently recovered phase evolution of each comb line using the method described in [25], together with the phase noise difference between a pair of the comb lines after back-toback and $150 \mathrm{~km}$ fiber transmission. To obtain the differential phase offset, we choose the phase of the master channel as a reference and subtract this phase estimate from the estimated phase drift of other line. 


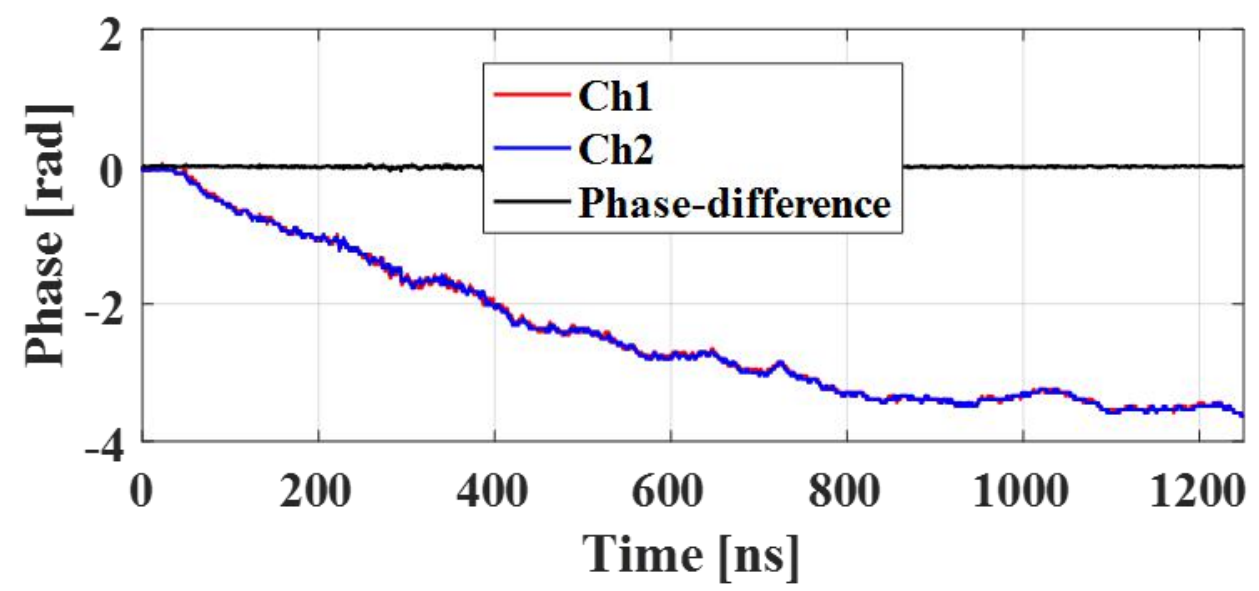

(a)

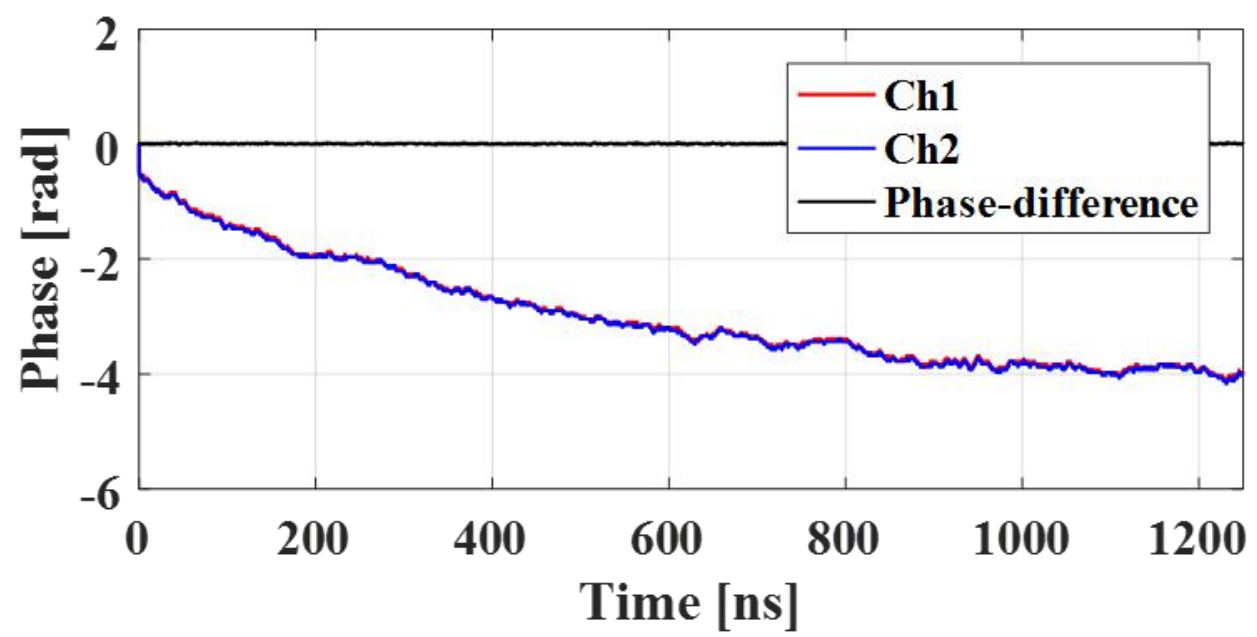

(b)

Fig. 9. Phase evolution for $40 \mathrm{GHz}$-spaced two channels after (a) back-to-back and (b) $150 \mathrm{~km}$ fiber transmission, together with the phase-difference between them.

As can be seen in Fig. 9, the curves for the phases extracted individually are similar and closely follow each other, even after fiber transmission up to $150 \mathrm{~km}$ in our experiments. This demonstrates that the use of optical frequency combs at the transmitter and receiver preserves the relative optical phase relationship between the comb lines after coherent detection in comb-based OFDM systems. Therefore, the high degree of correlated phase evolution among the OFDM bands provides the possibility to reduce the number of pilot subcarriers used for phase estimation via master-slave carrier phase tracking technique, leading to a reduced overhead for OFDM-based superchannel transmission systems enabled by optical frequency combs. Furthermore, it can also be observed from Fig. 9 that the differential phase offset is smaller and drifts much slower in magnitude than the actual common phase fluctuations derived directly from the transmitter and LO laser sources. This shows that the differential phase offset is a scaled-down version of the common phase noise among the OFDM bands. For the back-to-back and $150 \mathrm{~km}$ fiber transmission cases considered in Fig. 9, it can also be 
observed that the differential phases are highly correlated. This altogether confirms our hypothesis in Section 2 that the phase fluctuation is a common phase noise impairment among the comb lines, which enables the master-slave carrier phase tracking technique in this work.

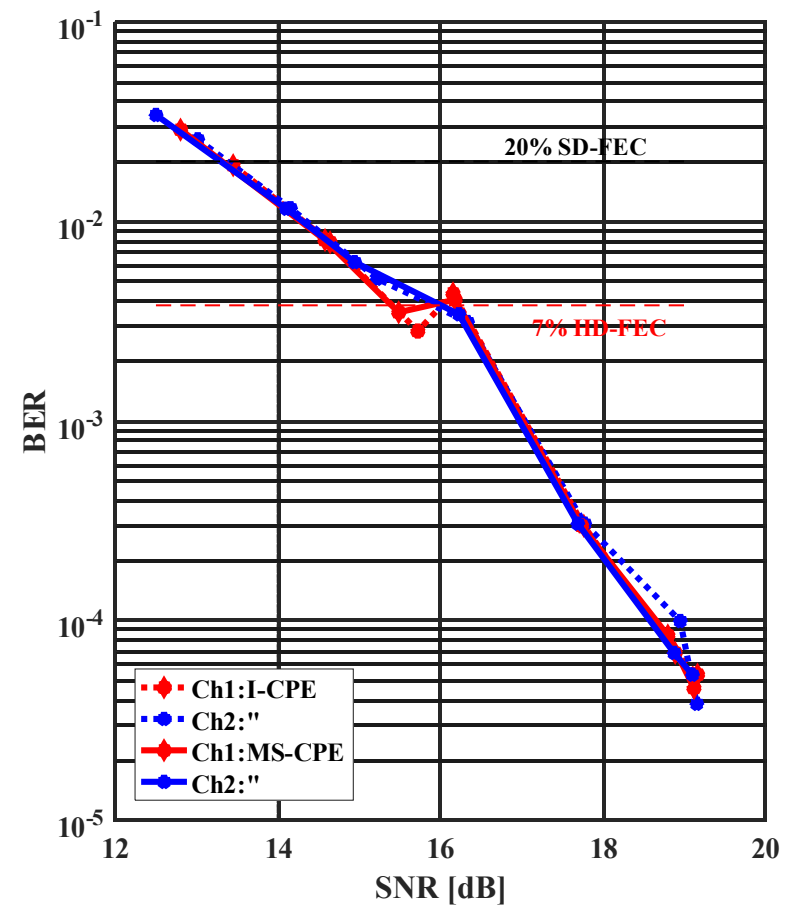

(a)

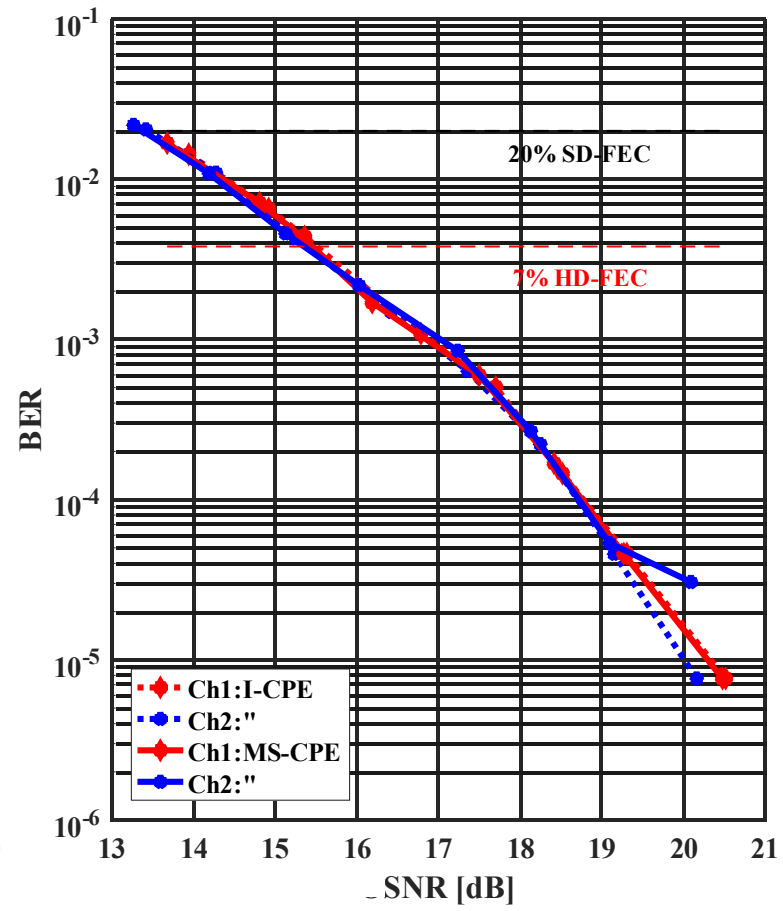

(b)

Fig. 10. BER as a function of SNR values obtained after (a) back-to-back and (b) $150 \mathrm{~km}$ transmission. I-CPE: independent carrier phase estimation; MS-CPE: master-slave carrier phase estimation.

Next, we experimentally study the performance of the master-slave carrier phase estimation (MS-CPE) technique designed in Section 2 and evaluated using simulation in Section 4. As the performance of the conventional independent carrier phase estimation (I-CPE) method is unaffected by the differential phase offset, we use the I-CPE method as a benchmark to evaluate the performance of our proposed master-slave joint-carrier phase tracking scheme. In Fig. 10(a) and (b), we plot the BER obtained as a function of SNR values after back-toback and $150 \mathrm{~km}$ fiber transmission. The back-to-back configuration without a fiber transmission link serves to distinguish penalties coming from the comb source from the fiber link by comparing performance results of both configurations. In Fig. 10(a) and (b), the SNR is calculated using the corresponding recovered data. It can be seen from Fig. 10(a) and (b) that the performance of the proposed master-slave joint-carrier phase tracking scheme closely matches the conventional performance when the carrier phase recovery algorithm is applied to each channel separately. In Fig. 11, we plot the BER performance as a function of OSNR and launch power values after $150 \mathrm{~km}$ fiber transmission distances. Here, the data from the 
received optical spectrum is used to find the corresponding OSNR values. In spite of few fluctuations observed in the BER performance shown in Fig. 11, the results still demonstrate the feasibility of joint-carrier phase tracking techniques. It can however be observed that the system performance is limited due to higher nonlinear distortions incurred as power level and fiber length increases. In this context, larger penalties can be expected for longer fiber transmission distances due to nonlinear phase noise accumulation during signal propagation. As the experiments are limited to short fiber lengths due to inadequate experimental resources, it is worth mentioning here that the differential phase offset discussed previously could not be observed for the results obtained. In this case, these results indicate that jointcarrier phase tracking technique does not significantly impair performance for systems with shorter fiber lengths, and can therefore be suitably applied for carrier phase recovery of higher-order modulation formats in short-reach coherent optical superchannel communication systems enabled by optical frequency combs.

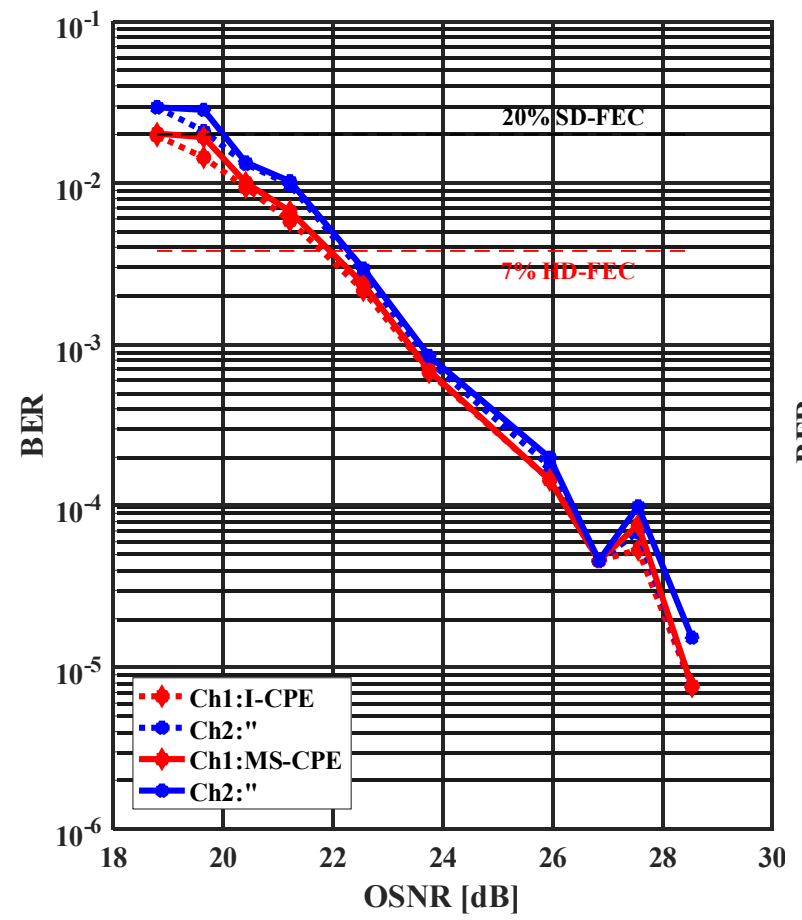

(a)

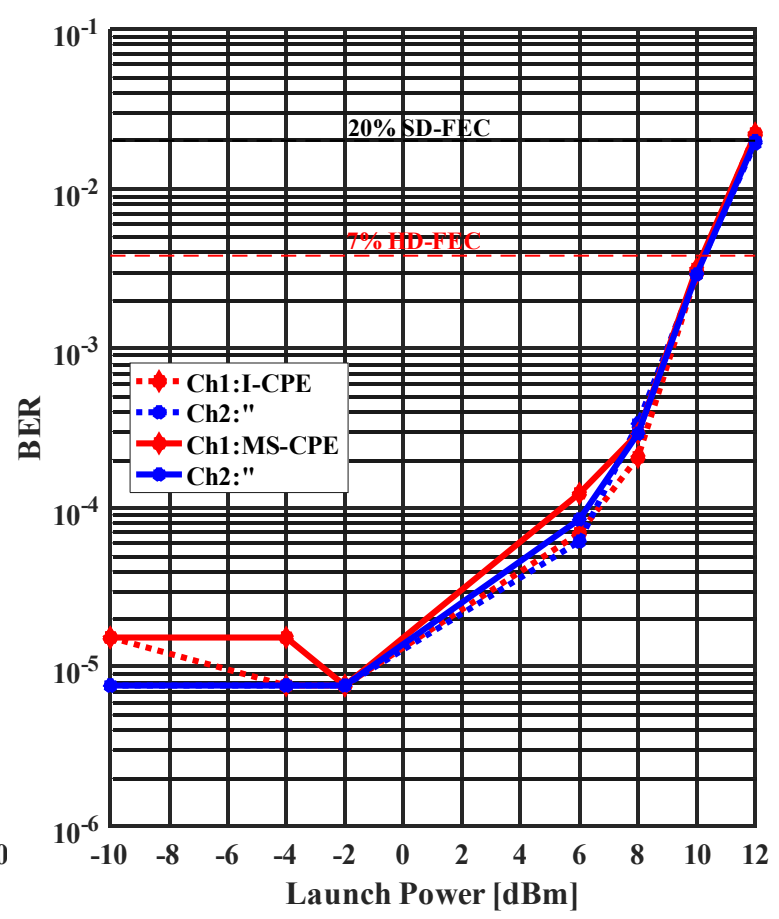

(b)

Fig. 11. BER as a function of (a) OSNR and (b) launch power values respectively after $150 \mathrm{~km}$ fiber transmission distances. I-CPE: independent carrier phase estimation; MS-CPE: master-slave carrier phase estimation.

\section{CONCLUSION}

In this work, utilizing the phase coherence provided by optical frequency combs, we have designed a carrier phase tracking scheme with lower overhead and computational complexity for OFDM-based spectral superchannel transmission systems. The proposed master-slave 
joint-carrier phase tracking technique show favorable performance for extensive simulation of OFDM-based superchannel that had 5 bands spaced at $50 \mathrm{GHz}$ with zero guard-band. The simulation is conducted using four different M-QAM (M = 4, 8, 16, and 32) modulation formats with laser and nonlinear phase noise effects after $7000 \mathrm{~km}, 4000 \mathrm{~km}, 3000 \mathrm{~km}$ and $2000 \mathrm{~km}$ SSMF transmission distances respectively. The simulation results show that there exist differential phase offset among OFDM-bands whose impact is dependent on the modulation, the channel count and fiber length. Consequently, the differential phase offset degrades joint-carrier phase tracking performance of outer sub-bands than the inner subbands. Finally, we demonstrate experimentally the feasibility performance of the designed master-slave carrier phase tracking technique using an OFDM-based superchannel with 2 OFDM-bands spaced at $40 \mathrm{GHz}$-spaced and each modulated with 16QAM signal format up to $150 \mathrm{~km}$ fiber transmission distance. Besides greatly improving the spectral efficiency, the phase-locked carriers originating from the optical frequency comb provides alternative means to reduce the overhead of pilot-subcarrier phase estimation in OFDM systems.

\section{ACKNOWLEDGMENT}

This work was supported by the National Natural Science Foundation of China (No. 61471088).

\section{REFERENCES}

[1] E. Agrell et al., "Roadmap of optical communications," J. Opt., vol. 18, no. 6, pp. 1-40, 2016.

[2] S. Chandrasekhar and X. Liu, "OFDM based superchannel transmission technology," J. Light. Technol., vol. 30, no. 24, pp. 3816-3823, 2012.

[3] X. Liu, S. Chandrasekhar, and P. J. Winzer, "Digital signal processing enabling multi-Tb/s superchannel transmission: an overview of recent advances in DSP-enabled superchannels," IEEE Signal Process. Mag., vol. 31, no. 2, pp. 16-24, 2014.

[4] P. J. Winzer and D. T. Neilson, "From scaling disparities to integrated parallelism : a decathlon for a decade," $J$. Light. Technol., vol. 35, no. 5, pp. 1099-1115, 2017.

[5] F. P. G. Uiomar, A. C. Arena, G. B. Osco, L. B. Ertignono, P. Torino, and C. Duca, "Nonlinear mitigation on subcarrier-multiplexed PM-16QAM optical systems," Opt. Express, vol. 25, no. 4, pp. 4069-4076, 2017.

[6] M. D. Feuer, L.E. Nelson, X. Zhou, S.L. Woodward, R. Isaac, B. Zhu, T.F. Taunay, M. Fishteyn, J.M. Fini, and M.F. Yan, "Joint digital signal processing receivers for spatial superchannels," IEEE Photonics Technol. Lett., vol. 24, no. 21, pp. 1957-1960, 2012.

[7] G. Bosco, V. Curri, A. Carena, P. Poggiolini, and F. Forghieri, "On the performance of Nyquist-WDM terabit superchannels based on PM-BPSK, PM-QPSK, PM-8QAM or PM-16QAM subcarriers," J. Light. Technol., vol. 29, no. 1, pp. 53-61, 2011.

[8] J. Pan, C. Liu, T. Detwiler, A. J. Stark, Y. H. Hsueh, and S. E. Ralph, "Inter-channel crosstalk cancellation for Nyquist-WDM superchannel applications,” J. Light. Technol., vol. 30, no. 24, pp. 3993-3999, 2012.

[9] D. Hillerkuss, R. Schmogrow, M. Meyer, S. Wolf, M. Jordan, P. Kleinow, N. Lindenmann, P.C. Schindler, A Melikyan, X. Yang, and S. Ben-Ezra, "Single-laser 32.5 Tbit/s Nyquist WDM transmission," J. Opt. Commun. Netw., vol. 4, no. 10, pp. 715-723, 2012.

[10] S. Yao, S. Fu, J. Li, M. Tang, and P. Shum, "Phase noise tolerant inter-carrier-interference cancellation for WDM superchannels with sub-Nyquist channel spacing," Opt. Express, vol. 21, no. 18, pp. 21569-21578, 2013.

[11] T. Xu, Xu, G. Liga, D. Lavery, B.C. Thomsen, S.J. Savory, R.I. Killey, and P. Bayvel, "Equalization enhanced phase noise in Nyquist-spaced superchannel transmission systems using multi-channel digital back-propagation," Sci. Rep., vol. 5, no. 13990, pp. 1-13, 2015. 
[12] L. Lundberg, M. Karlsson, A. Lorences-Riesgo, and M. Mazur, "Frequency comb-based WDM transmission systems enabling joint signal processing," Appl. Sci., vol. 8, no. 718, pp. 1-25, 2018.

[13] F. K. Deynu, E. W. Akpari, and C. Akama, "Phase noise effects on the performance of joint carrier phase recovery algorithms in phase-locked WDM superchannel transmission systems," Opt. Fiber Technol., vol. 54, no. 102104, pp. $1-11,2020$.

[14] F. K. Deynu, B. Xu, and E. W. Akpari, "Design of feedforward master-slave carrier phase recovery in frequency comb-based superchannel coherent transmission systems with nonlinear phase noise," Opt. Commun., vol. 459, no. 124898, pp. 1-14, 2020.

[15] G. Bosco, A. Carena, V. Curri, P. Poggiolini, and F. Forghieri, "Performance limits of Nyquist-WDM and COOFDM in high-speed PM-QPSK systems," IEEE Photonics Technol. Lett., vol. 22, no. 15, pp. 1129-1131, 2010.

[16] S. Park, "Effect of the phase-error of local oscillators in digital back-propagation," IEEE Photonics Technol. Lett., vol. 27, no. 4, pp. 363-366, 2015.

[17] X. Yi, N. K. Fontaine, R. P. Scott, and S. J. B. Yoo, "Tb/s coherent optical OFDM systems enabled by optical frequency combs," J. Light. Technol., vol. 28, no. 14, pp. 2054-2061, 2010.

[18] E. F. Mateo, X. Zhou, S. Member, G. Li, and S. Member, "Selective Post-Compensation of Nonlinear Impairments in Polarization-Division Multiplexed WDM Systems with Different Channel Granularities," IEEE J. Quantum Electron., vol. 47, no. 1, pp. 109-116, 2011.

[19] Y.-K. Huang, E. Ip, Z. Wang, M.-F. Huang, Y. Shao, and T. Wang, "Transmission of spectral efficient superchannels using all-optical OFDM and digital coherent receiver technologies," J. Light. Technol., vol. 29, no. 24, pp. 3838-3844, 2011.

[20] G. Colavolpe, T. Foggi, E. Forestieri, and M. Secondini, "Impact of phase noise and compensation techniques in coherent optical systems,” J. Light. Technol., vol. 29, no. 18, pp. 2790-2800, 2011.

[21] S. L. Jansen, B. Spinnler, I. Morita, S. Randel, and H. Tanaka, “100GbE : QPSK versus OFDM,” Opt. Fiber Technol., vol. 15, pp. 407-413, 2009.

[22] A. Barbieri, G. Colavolpe, T. Foggi, E. Forestieri, and G. Prati, "OFDM versus single-carrier transmission for 100 Gbps optical communication," J. Light. Technol., vol. 28, no. 17, pp. 2537-2551, 2010.

[23] Xingwen Yi, William Shieh, and Yiran Ma, "Phase noise effects on high spectral efficiency coherent optical OFDM transmission," J. Light. Technol., vol. 26, no. 10, pp. 1309-1316, 2008.

[24] X. Yi et al., "Digital coherent superposition of optical OFDM subcarrier pairs with Hermitian symmetry for phase noise mitigation," Opt. Express, vol. 22, no. 11, pp. 13454-13459, 2014.

[25] X. Yi, W. Shieh, and Y. Tang, "Phase estimation for coherent optical OFDM," IEEE Photonics Technol. Lett., vol. 19, no. 12, pp. 919-921, 2007.

[26] S. T. Le, T. Kanesan, E. Giacoumidis, N. J. Doran, and A. D. Ellis, "Quasi-pilot aided phase noise estimation for coherent optical OFDM systems,” IEEE Photonics Technol. Lett., vol. 26, no. 5, pp. 504-507, 2014.

[27] X. Zhang, J. Li, C. Li, M. Luo, H. Li, Z. He, Q. Yang, C. Lu, Z. Li, "Pilot-based blind phase estimation for coherent optical OFDM system," Opt. Express, vol. 22, no. 19, pp. 22888-22893, 2014.

[28] H. Ren, J. Cai, J. Lu, H. Wen, L. Xue, Y. Qin, S. Guo, S. Zhou, and W. Hu, "Novel iteration-free blind phase noise estimation for coherent optical OFDM," Chinese Opt. Lett., vol. 12, no. 12, pp. 1-5, 2014.

[29] S. T. Le, Le, P.A. Haigh, A.D. Ellis, and S.K. Turitsyn, "Blind phase noise estimation for CO-OFDM transmissions," J. Light. Technol., vol. 34, no. 2, pp. 745-753, 2015.

[30] M. Pan, X. Zhang, J. Li, and Z. Li, "Data-aided linear fitting blind phase estimation method for coherent optical OFDM system,” Photonic Netw. Commun., vol. 31, no. 2, pp. 316-320, 2016.

[31] T. Bo and C.-K. Chan, "Common phase error estimation in coherent optical OFDM systems using best-fit bounding box," Opt. Express, vol. 24, no. 21, pp. 23707-23718, 2016.

[32] X. Fang, C. Yang, T. Zhang, and F. Zhang, "Orthogonal basis expansion-based phase noise suppression for PDM CO-OFDM system,” IEEE Photonics Technol. Lett., vol. 26, no. 4, pp. 376-379, 2014

[33] X. Hong, X. Hong, and S. He, "Linearly interpolated sub-symbol optical phase noise suppression in CO-OFDM system," Opt. Express, vol. 23, no. 4, pp. 4691-4702, 2015.

[34] Q. Fan, J. He, M. Chen, J. Liu, and L. Chen, "Low-complexity phase noise compensation approach for CO-OFDM systems," IEEE Photonics Technol. Lett., vol. 28, no. 21, pp. 2323-2326, 2016.

[35] X. Hong, X. Hong, and S. He, "Low-complexity optical phase noise suppression in CO-OFDM system using recursive principal components elimination," Opt. Express, vol. 23, no. 18, pp. 24077-24087, 2015.

[36] W. Bao, M. Bi, S. Xiao, J. Fang, T. Huang, and Y. Zhang, "Lagrange interpolation based extended Kalman filter for 
Journal of Microwaves, Optoelectronics and Electromagnetic Applications, Vol. 20, No. 1, March 2021

DOI: http://dx.doi.org/10.1590/2179-10742021v20i1965

phase noise suppression in CO-OFDM system,” Opt. Commun., vol. 435, pp. 221-226, 2019.

[37] J. Yuan, W. Bi, W. Bi, and L. Xu, "A novel two-stage phase noise estimation algorithm for coherent optical OFDM systems," Optik (Stuttg)., vol. 124, no. 24, pp. 7053-7055, 2013.

[38] Y. Ha and W. Chung, "Non-data-aided phase noise suppression scheme for CO-OFDM systems," IEEE Photonics Technol. Lett., vol. 25, no. 17, pp. 1703-1706, 2013.

[39] T. T. Nguyen, S. T. Le, M. Wuilpart, and P. Megret, "Experimental Demonstration of a Low-Complexity Phase Noise Compensation for CO-OFDM Systems," IEEE Photonics Technol. Lett., vol. 30, no. 16, pp. 1467-1470, 2018.

[40] A. J. Lowery and L. B. Du, "Optical orthogonal division multiplexing for long haul optical communications: A review of the first five years," Opt. Fiber Technol., vol. 17, no. 5, pp. 421-438, 2011.

[41] L. Lundberg, M. Mazur, A. Lorences-Riesgo, M. Karlsson, and P. A. Andrekson, "Joint carrier recovery for DSP complexity reduction in frequency comb-based superchannel transceivers," in European Conference on Optical Communication (ECOC), 2017, pp. 1-3.

[42] X. Chen, J. He, D. Che, and W. Shieh, "Low complexity phase noise compensation for multiband coherent optical OFDM," in OptoElectronics and Communication Conference (OECC)/Australian Conference on Optical Fibre Technology (ACOFT), 2014, pp. 910-912.

[43] I. Fatadin, D. Ives, and S. J. Savory, "Blind equalization and carrier phase recovery in a 16-QAM optical coherent system," J. Light. Technol., vol. 27, no. 15, pp. 3042-3049, 2009.

[44] P. J. Winzer, A. H. Gnauck, C. R. Doerr, M. Magarini, and L. L. Buhl, "Spectrally efficient long-haul optical networking using 112-Gb/s polarization-multiplexed 16-QAM,” J. Light. Technol., vol. 28, no. 4, pp. 547-556, 2010 .

[45] Y. Mori, C. Zhang, and K. Kikuchi, "Novel configuration of finite-impulse-response filters tolerant to carrier-phase fluctuations in digital coherent optical receivers for higher- order quadrature amplitude modulation signals," Opt. Express, vol. 20, no. 24, pp. 26236-26251, 2012.

[46] R. Noé, T. Pfau, M. El-Darawy, and S. Hoffmann, "Electronic Polarization Control Algorithms for Coherent Optical Transmission,” IEEE J. Sel. Top. Quantum Electron., vol. 16, no. 5, pp. 1193-1200, 2010.

[47] A. Sano, E. Yamada, H. Masuda, E. Yamazaki, T. Kobayashi, E. Yoshida, Y. Miyamoto, R. Kudo, K. Ishihara, and Y. Takatori, "No-guard-interval coherent optical OFDM for 100-Gb/s/ch long-haul transmission systems," J. Light. Technol., vol. 27, no. 16, pp. 3705-3713, 2009.

[48] M. S. Faruk and S. J. Savory, "Digital signal processing for coherent transceivers employing multilevel formats," $J$. Light. Technol., vol. 35, no. 5, pp. 1125-1141, 2017.

[49] J. C. Cartledge, F. P. Guiomar, F. R. Kschischang, G. Liga, and M. P. Yankov, "Digital signal processing for fiber nonlinearities," Opt. Express, vol. 25, no. 3, pp. 1916-1936, 2017. 